\title{
Localization and Localizability in Sensor and Ad-hoc Networks
}

\author{
Ph.D. Dissertation Defense
}

\section{Zheng Yang}

Advisor: Prof. Yunhao Liu

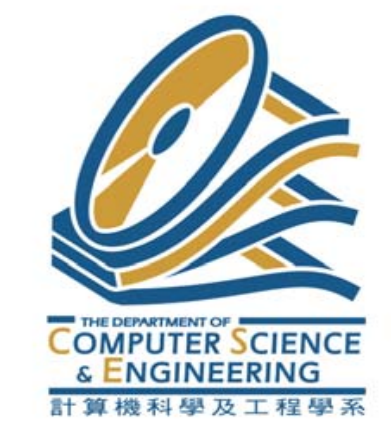

香港科技大學 HONG KONG UNIVERSITY OF SCIENCE \& TECHNOLOGY 


\section{(TT)}

"The success of a retail store depends on three factors: location, location, and location."

- anonymous

"So does wireless networking. "

— Zheng Yang 


\section{Outline}

$\square$ Introduction

$\square$ Localization

$\square$ Localizability

- Network Localizability

- Distributed Localizability Testing

- Node Localizability

$\square$ Conclusion and Future Study 


\section{What is a Sensor Node?}

$\square$ Sensor is a tiny electronic device with four major components

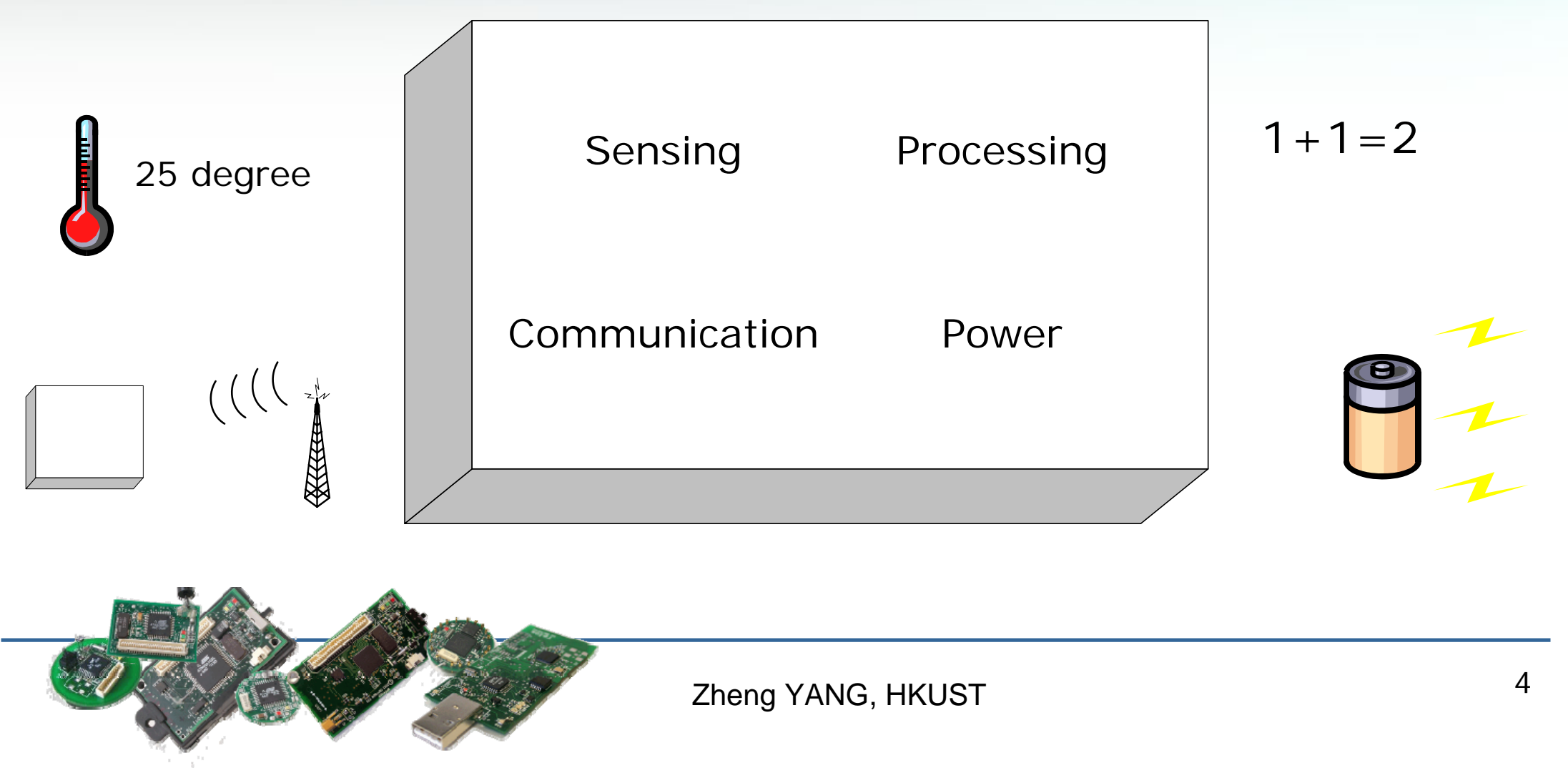




\section{What is a Sensor Node?}

$\square$ Different kinds of sensor nodes

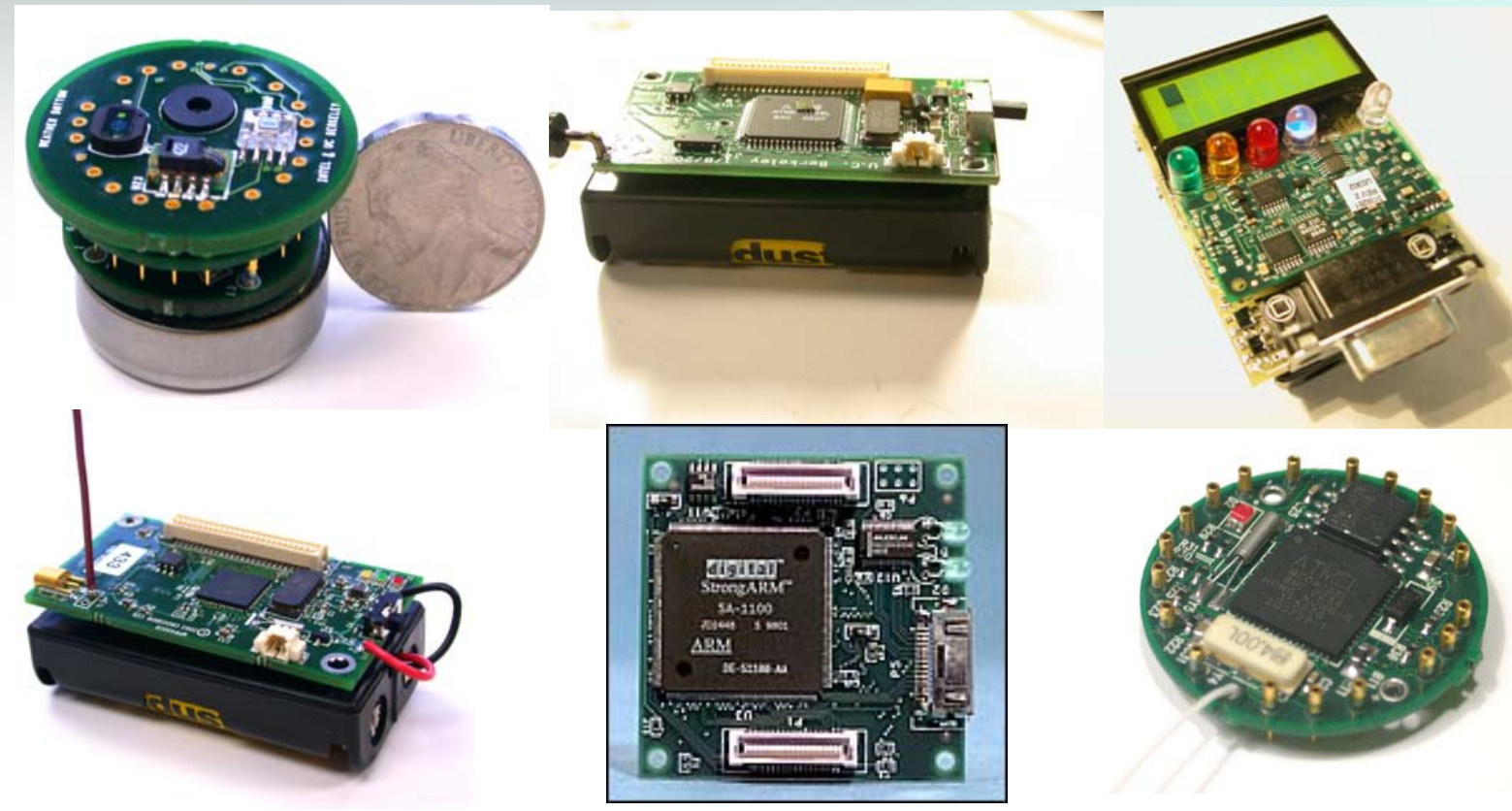




\section{What is a WSN?}

$\square$ Wireless Sensor Network (WSN)

a large amount, spatially distributed, and autonomous sensors

- cooperatively monitor physical world.

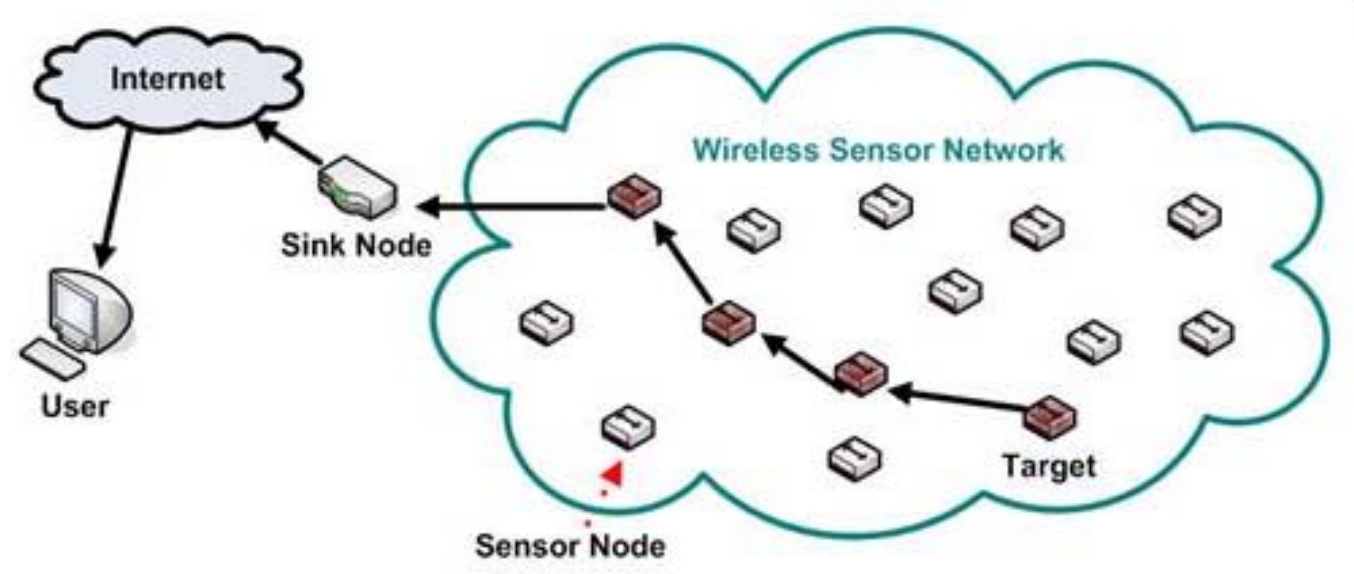




\section{What is a WSN?}

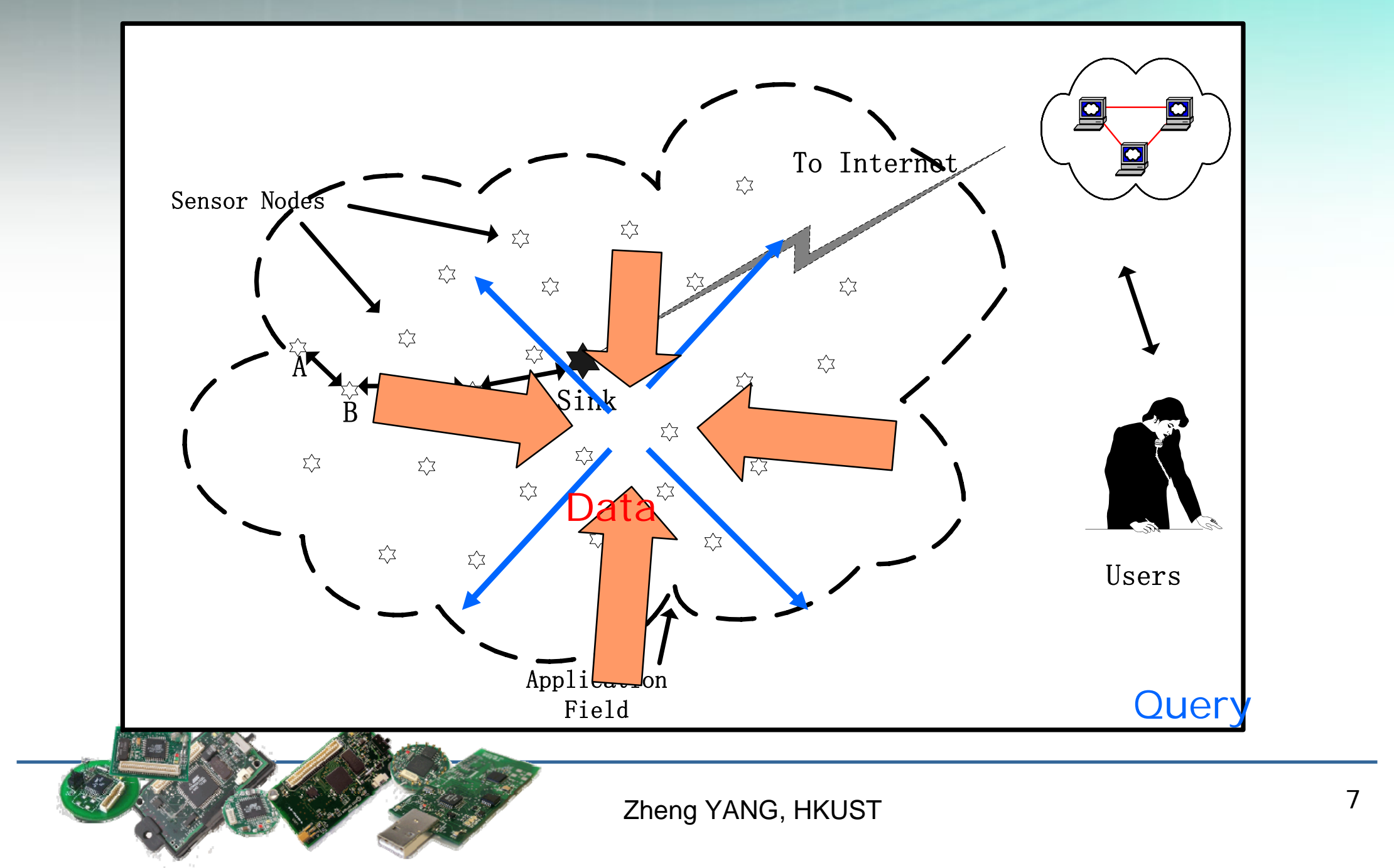




\section{WSN Applications}

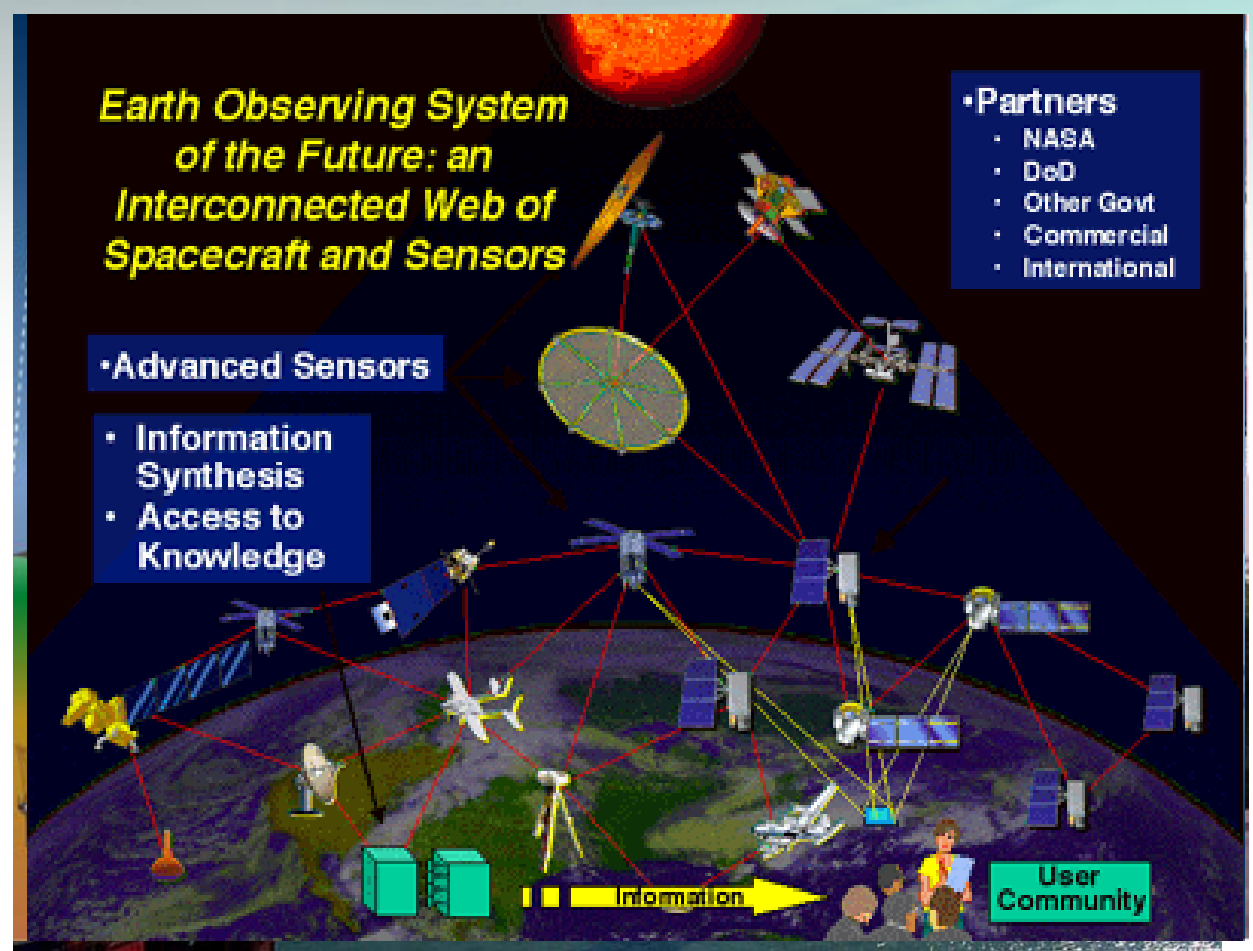




\section{Outline}

$\square$

$\square$ Localization

$\square$ Localizability

- Network Localizability

- Distributed Localizability Testing

- Node Localizability

$\square$ Conclusion and Future Study 


\section{Localization}

$\square$ Determine the locations of wireless devices in a network
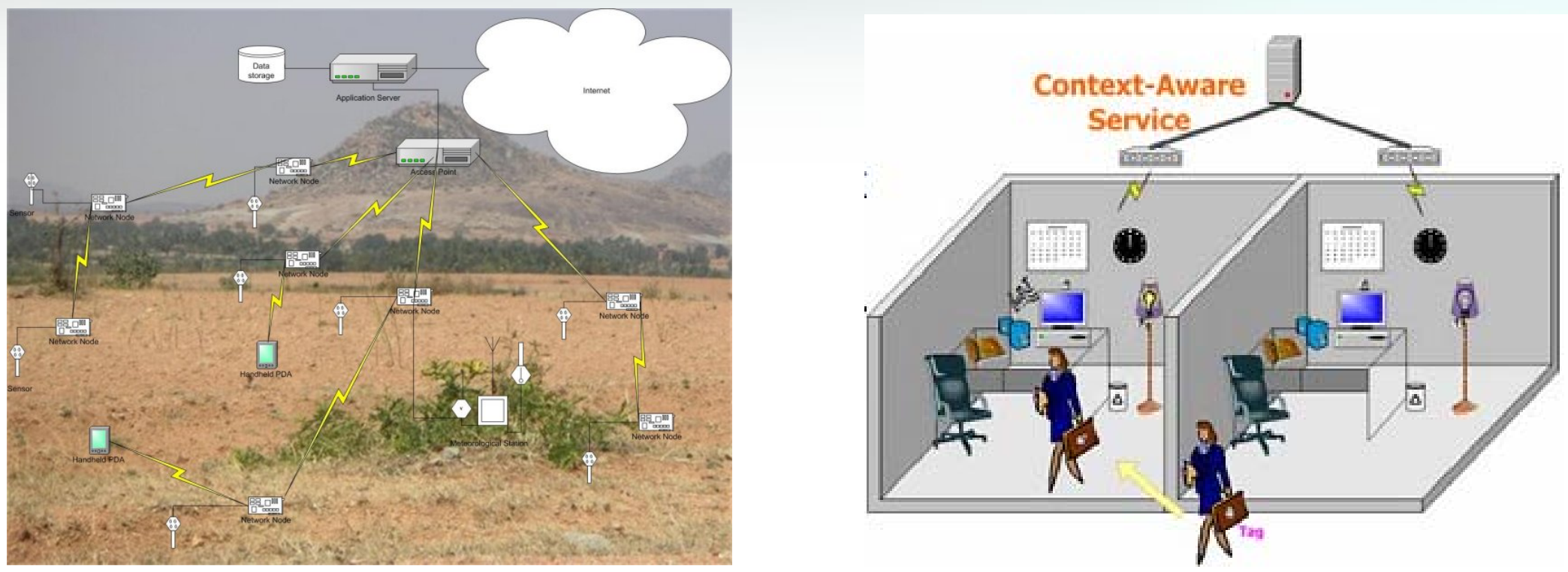


\section{Why Is Localization Important?}

$\square$ Wireless sensor networks

- Application

$\square$ Environmental monitoring, object tracking, ...

$\square$ "Sensing data without knowing the sensor location are meaningless." [IEEE Computer, Vol. 33, 2000]

- Localization aids other network functions

$\square$ Routing, topology control, clustering, ... 


\section{Why is Localization a Non-Trivial Problem?}

$\square$ Manual configuration

- Unscalable and sometimes impossible

$\square$ Why not use GPS?

- Increasing hardware costs

- Obstructions to GPS satellites

$\square$ Indoor

$\square$ Underground

- GPS accuracy (10-20 feet) poor for short range application
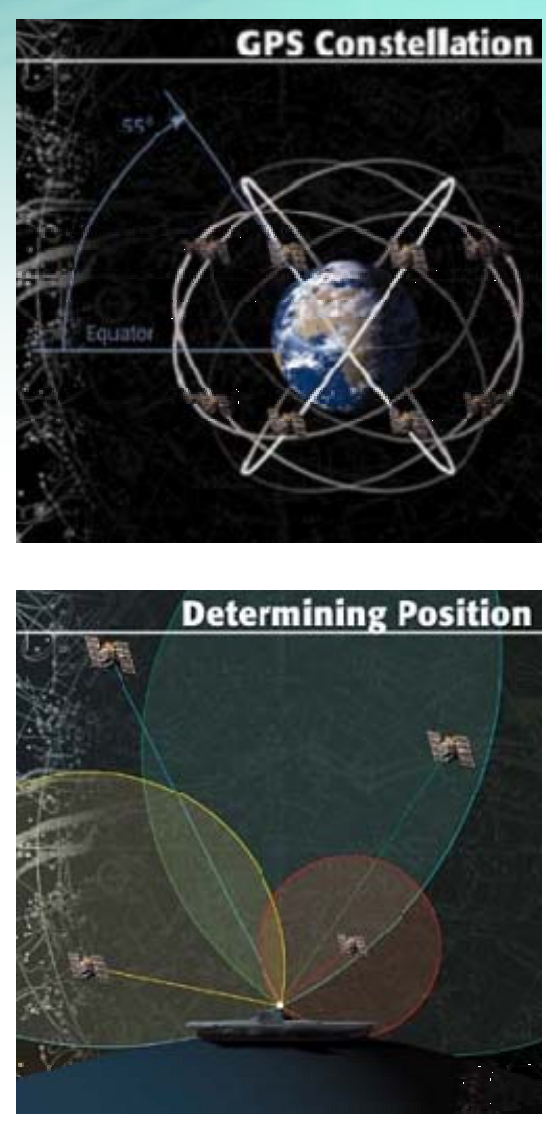


\section{Network Localization}

$\square$ Network Localization has been proposed for WSN

- Beacons

$\square$ special nodes at known locations

- Non-beacon nodes

$\square$ Determine locations by measuring geographic information to nearby nodes 


\section{Network Localization}

$\square$ Network localization consists of two steps

\section{Physical Measurement}

Fine-Grained

Coarse-Grained

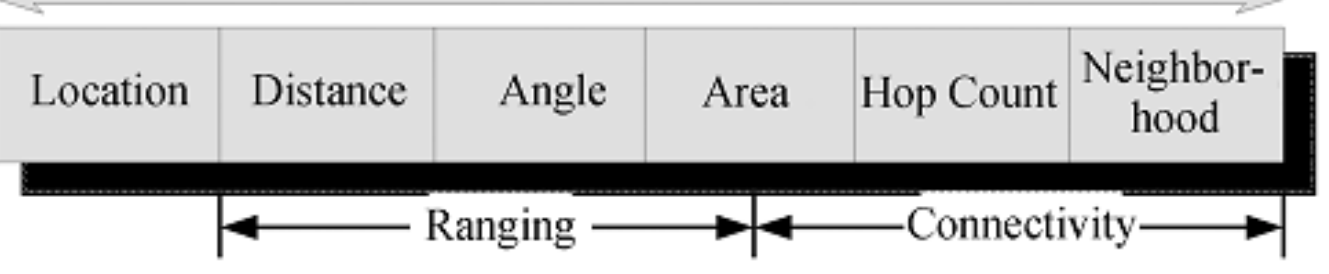

2. Location Computation

$\square$ This study focuses on range-based localization 


\section{Distance Measuring}

$\square$ Ranging techniques

- Radio Signal Strength (RSS)

- Time Difference of Arrival (TDoA)

$\square$ Ranging systems

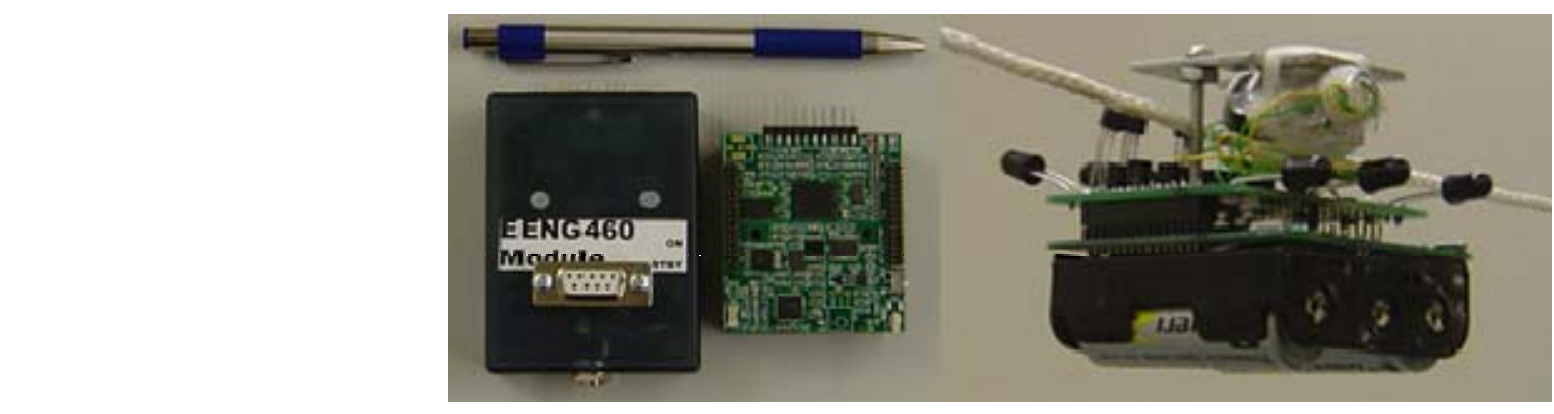

UCLA medusa mote

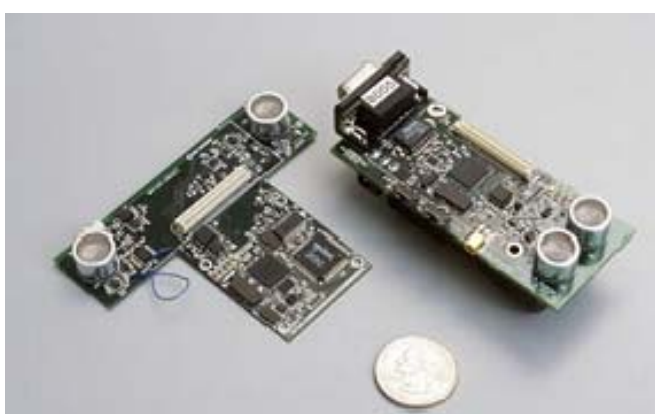

Yale XYZ mote

MIT Cricket mote 


\section{Distance Graph Model}

$\square$ Distance graph $\mathrm{G}_{\mathrm{N}}$ of a wireless network $\mathrm{N}$

- Vertices: wireless devices (e.g., laptops, PDAs, or sensor nodes)

- Edges: an edge connecting two vertices ( $\mathrm{i}$ and $\mathrm{j}$ ) if the distance $d(i, j)$ between corresponding nodes can be measured

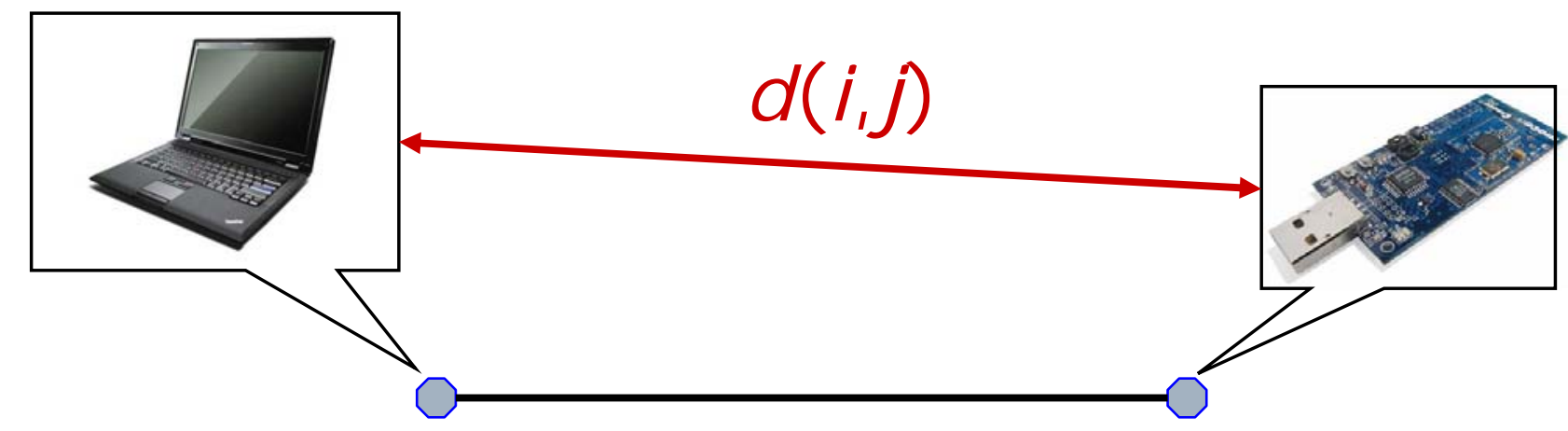




\section{Distance Graph Model}

\section{$\square$ Example}

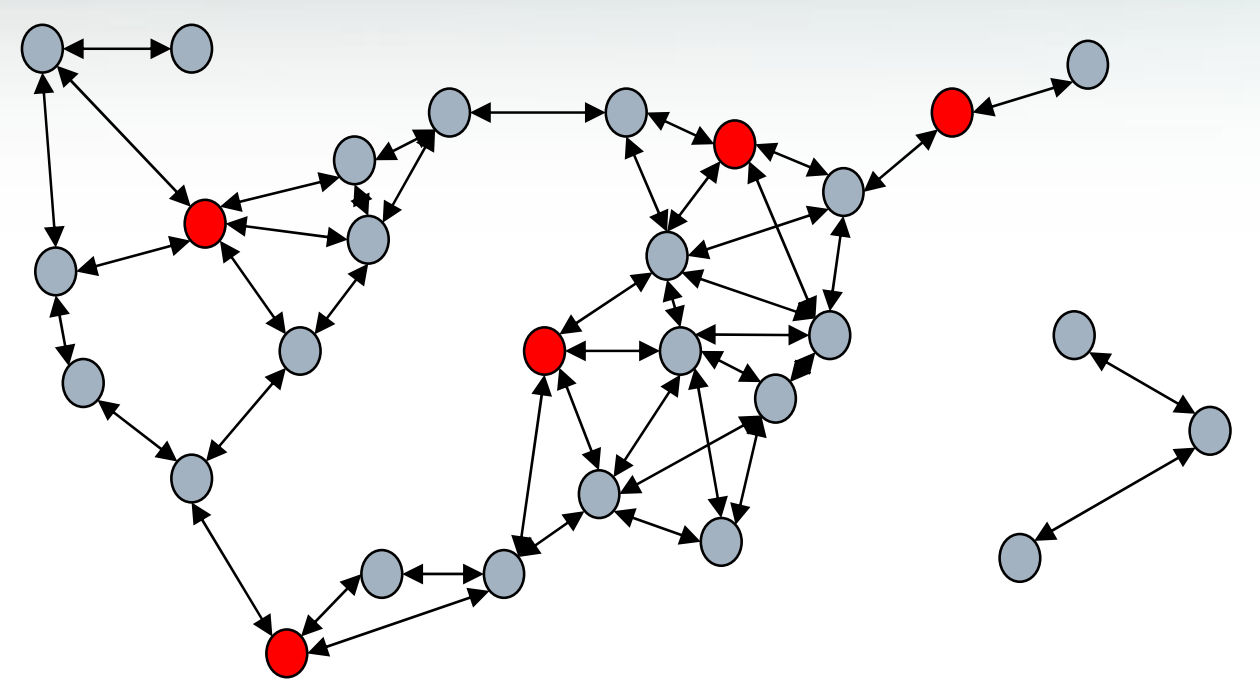

$\begin{array}{ll}\bigcirc & \text { node with known position (anchor) } \\ \longleftrightarrow \text { node with unknown position } \\ \end{array}$




\section{Location Computation}

$\square$ Trilateration

- Idea

$\square$ A location of an object can be determined if distances to

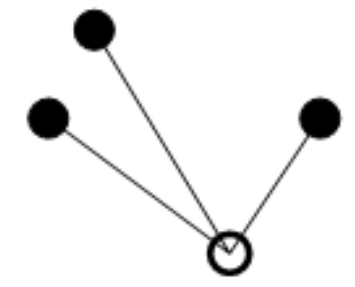
three anchors are known.

- Advantages

$\square$ Efficient

$\square$ Distributed

$\square$ Easy to implement

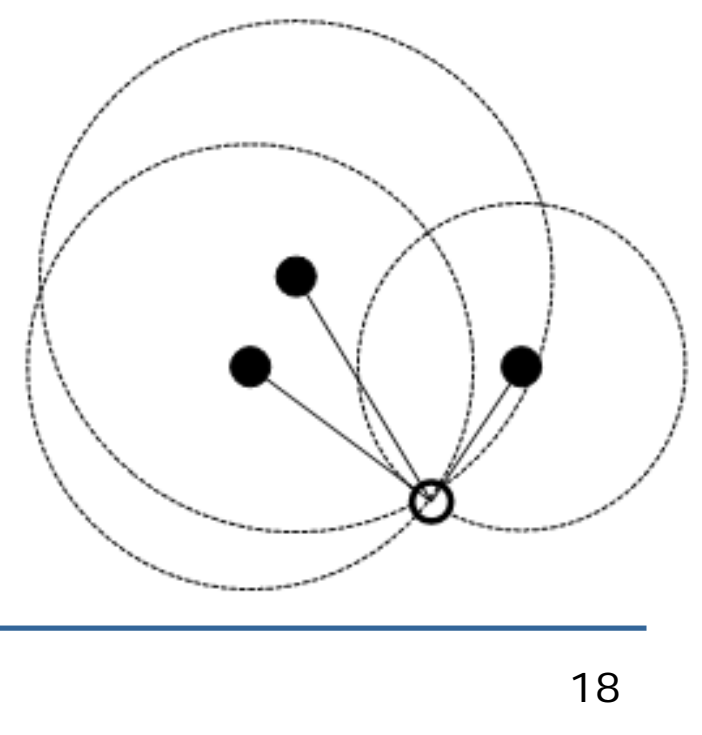




\section{Location Computation}

$\square$ Iterative trilateration

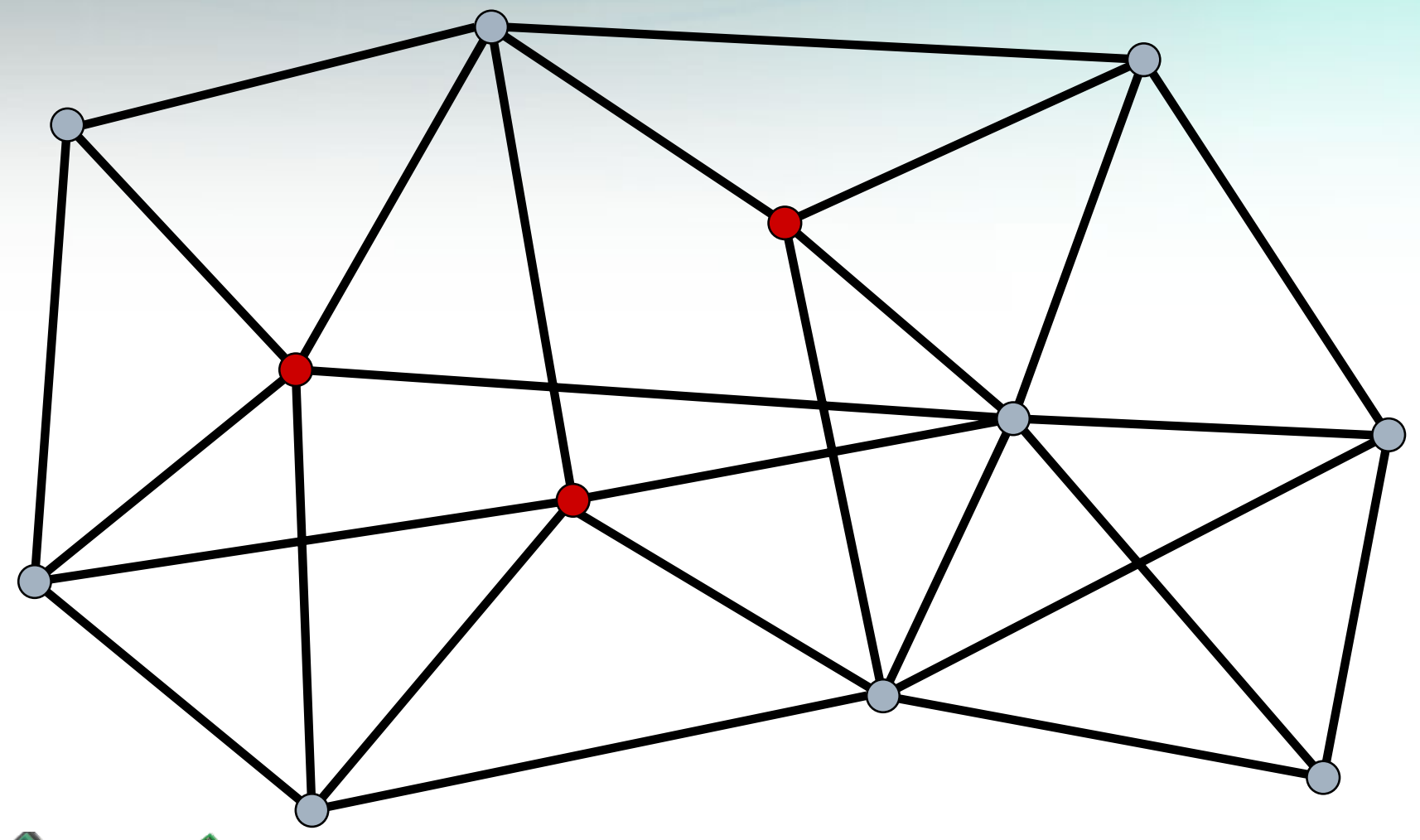




\section{Outline}

$\square$

$\square$ Localization

$\square$ Localizability

- Network Localizability

- Distributed Localizability Testing

- Node Localizability

$\square$ Conclusion and Future Study 


\section{Localizability}

\section{Definition}

A network is localizable

if it has a unique realization (or embedding) that respect the pairwise distance constraints and beacon locations in some metric space. 


\section{Localizability}

\section{$\square$ Localizability V.S Localization}

- If a network is NOT localizable, by no means it can be localized.

- If a network is localizable, it can be localized in theory (but may be computationally infeasible). 


\section{Conditions of Localizability}

$\square$ Sufficiency

What properties make a graph localizable?

$\square$ Necessity

- What properties a localizable graph has? 


\section{Localizability and Graph Rigidity [】]}
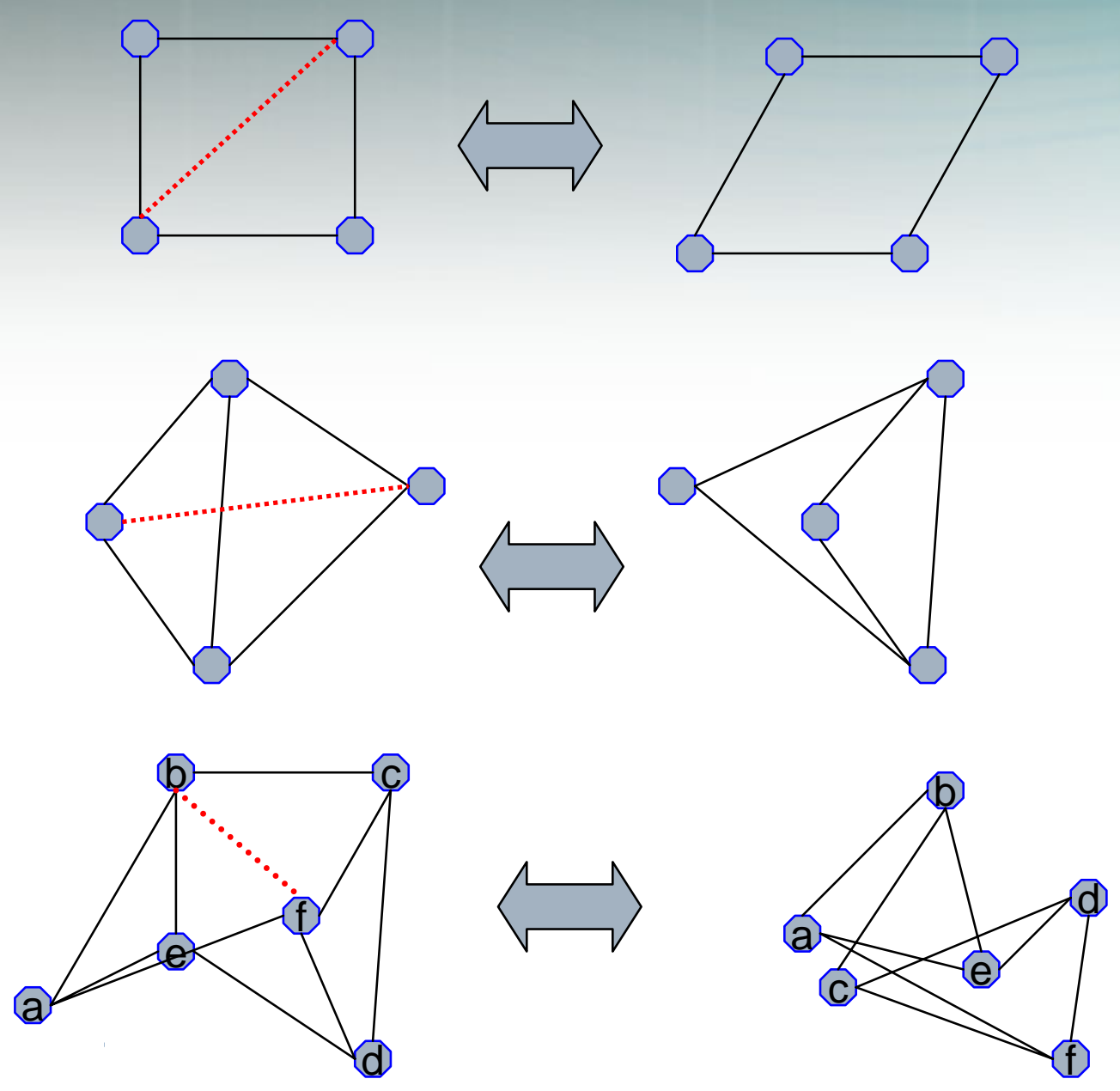

\section{Solution:}

G must be rigid.

G must be 3-connected.

$\mathrm{G}$ must be redundantly rigid: It must remain rigid upon removal of any single edge. 


\section{Localizability and Graph Rigidity (}

Global rigidity (by Jackson and Jordan, 2003)

A graph is generically globally rigid in 2D plane iff. it is 3-connected and redundantly rigid.

The necessary and sufficient condition of localizability.

Network localizability (Eren, 2004)

A network is localizable iff. its distance graph is globally rigid and it contains at least three beacons. 


\section{Localizability Test Algorithm}

$\square$ Network localizability can be tested

- Polynomial time to the graph size

$\square$ Rigidity: $O\left(n^{2}\right)$ by the pebble game algorithm by Jacobs and Hendrickson (1997)

$\square$ Redundant rigidity: $O\left(n^{2}\right)$ algorithm by Hendrickson (1991)

$\square$ 3-connectivity: $\mathrm{O}(\mathrm{n})$ algorithm by Tarjan (1972)

$\square$ So far, it seems ... 


\section{Outline}

$\square$

$\square$ Localization

$\square$ Localizability

- Network Localizability

- Distributed Localizability Testing

- Node Localizability

$\square$ Conclusion and Future Study 


\section{Difficulties of Localizability Test (}

$\square$ Global knowledge is needed

- Connectivity

- Rigidity

$\square$ Hard to design distributed approaches

- Trilateration as a compromise

- Nodes located by TRI are localizable

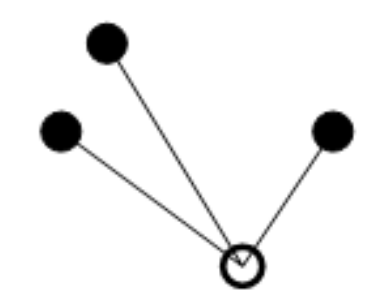




\section{Limitation of Trilateration}

$\square$ Only identify a subset of localizable networks (trilateration extension)

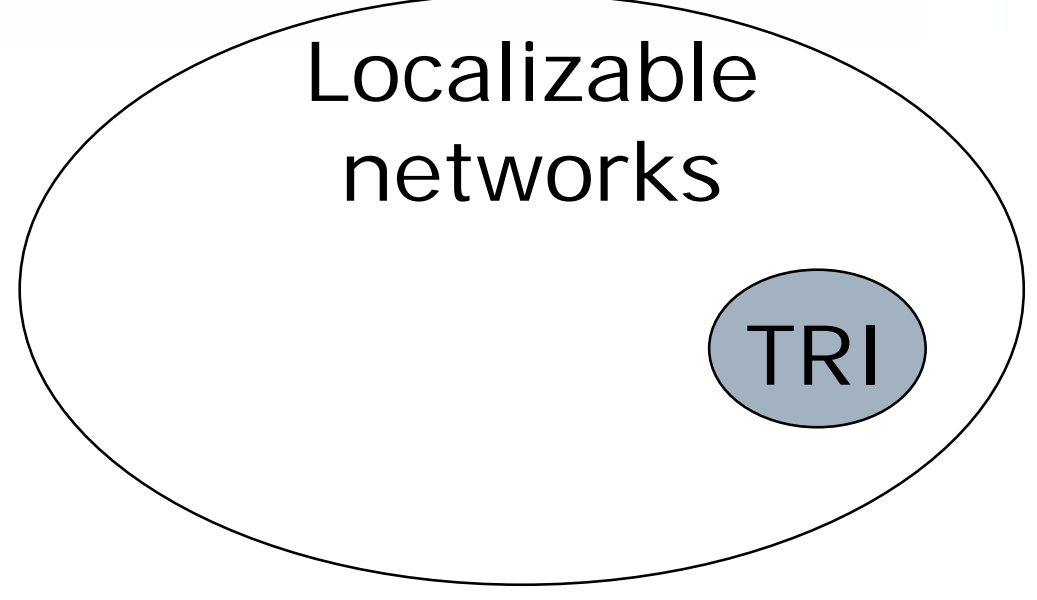




\section{Limitation of Trilateration}

$\square$ Fails to identify border nodes as localizable

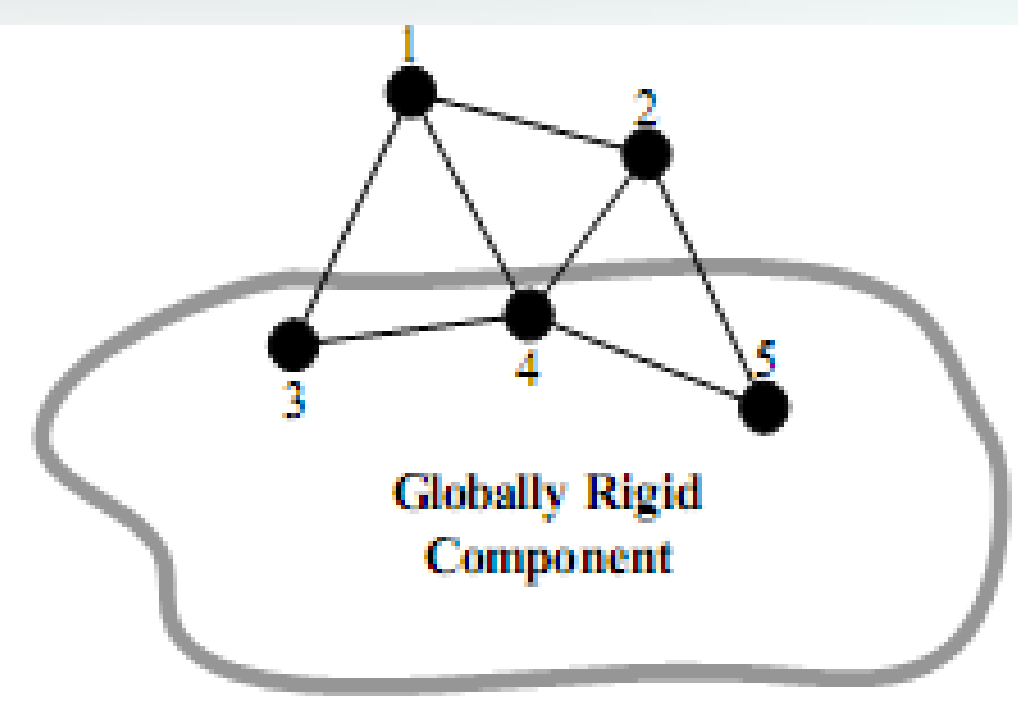




\section{Motivation}

$\square$ Motivate a novel approach for testing localizability

- Efficient

- Distributed

- Capable: identifying a larger number of localizable nodes than TRI

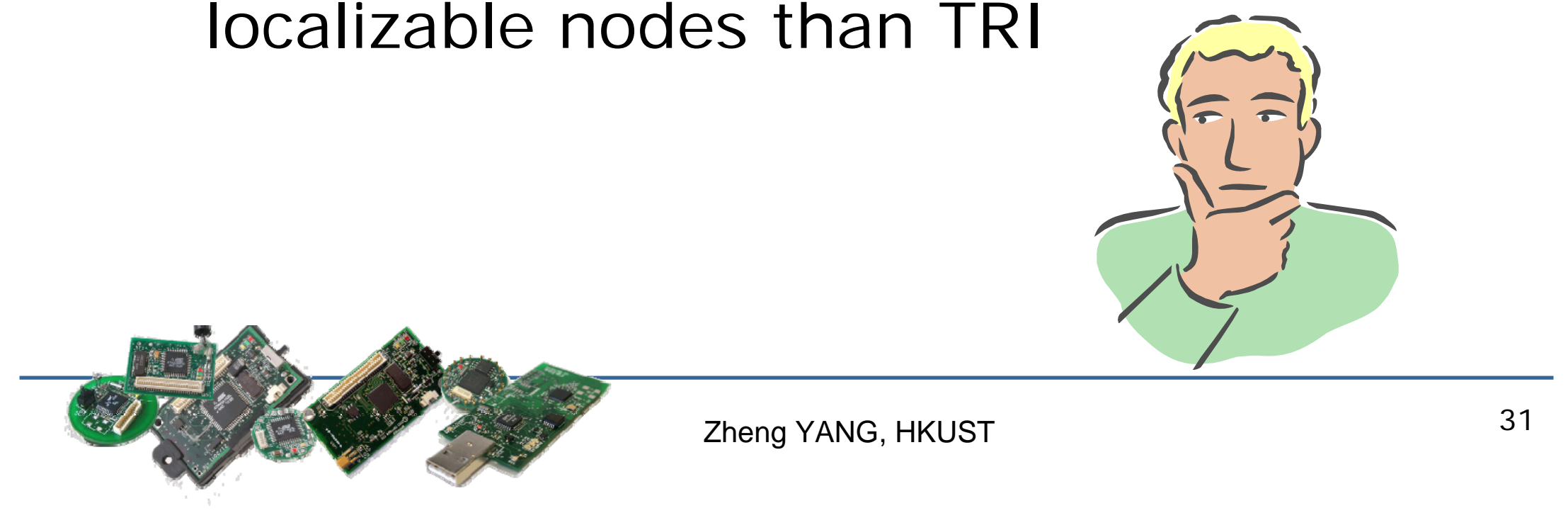




\section{Wheel graphs}

$\square$ A wheel graph $W_{n}$ consists of

- A hub vertex

- (n-1) rim vertices

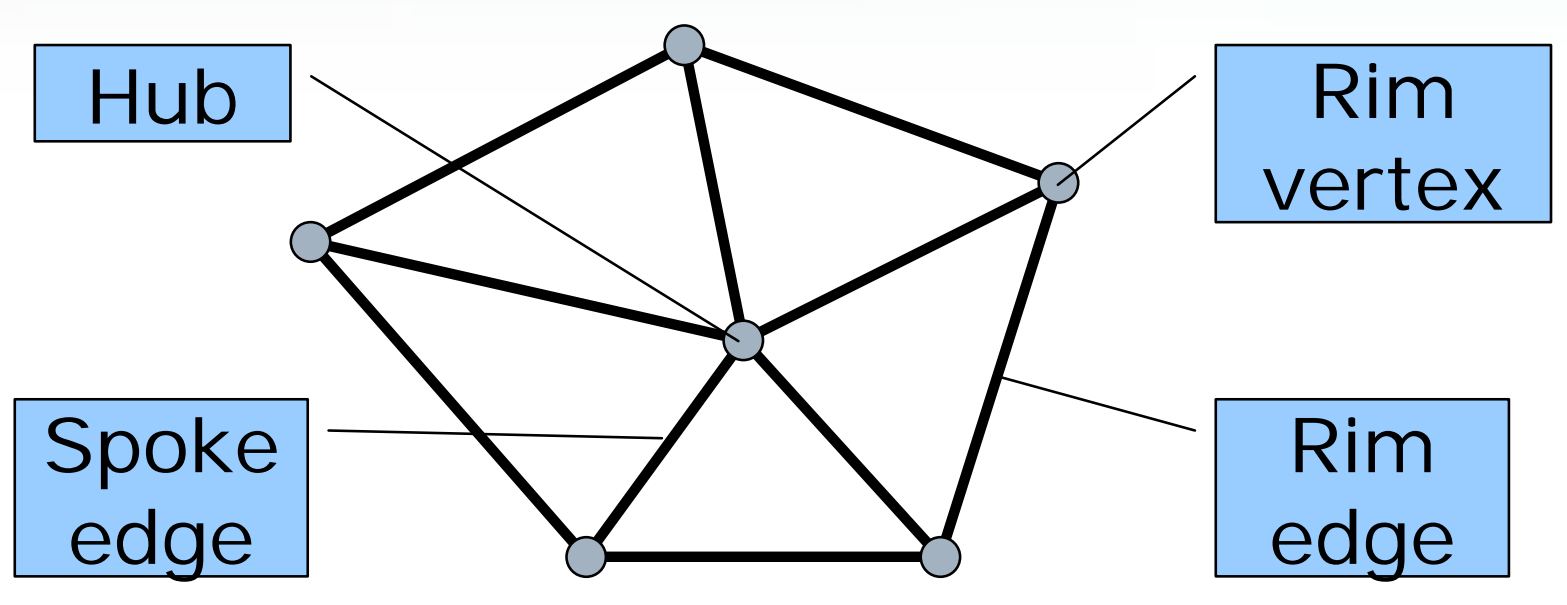




\section{Wheel graphs}

$\square$ Model 1-hop neighborhood abstract

- From the standpoint of the hub vertex

- All elements are in its 1-hop neighborhood

$\square$ vertices and edges

$\square$ Wheel graphs are globally rigid

- They are 3-connected and redundantly rigid.

I dentify localizable vertices based on the wheel instead of TRI! 


\section{Network-wide Localizability (I)}
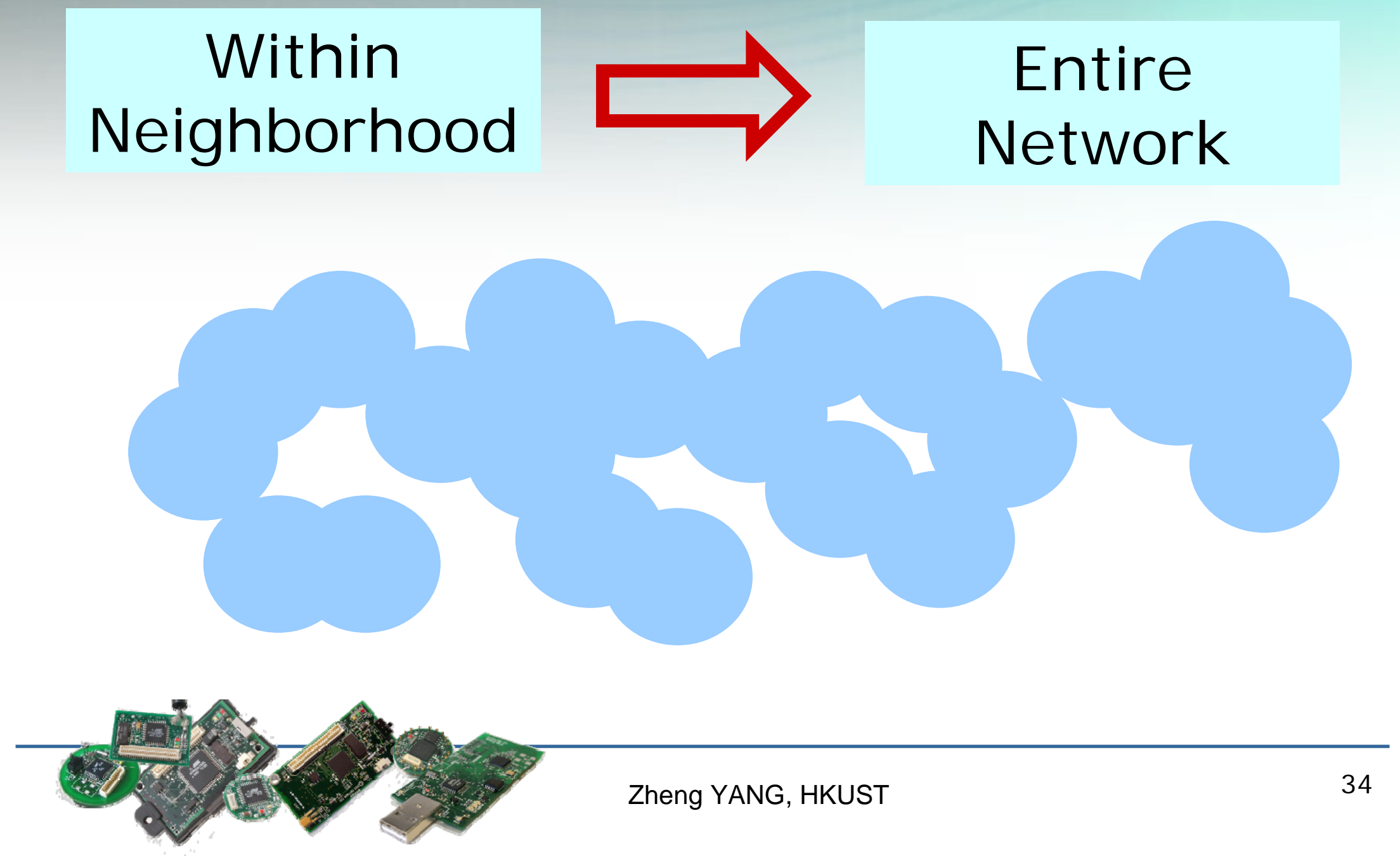


\section{Wheel Extension}

Definition 1.

A graph $\mathrm{G}$ is a wheel extension if there are

(a)three pairewise connected vertices, say $v_{1}$, $\mathrm{v}_{2}$, and $\mathrm{v}_{3}$; and

(b) an ordering of remaining vertices, say $v_{4}$, $v_{5}, v_{6}, \ldots$, such that any vertex $v_{i}$ is included in a wheel graph containing three early vertices in the sequence. 


\section{Wheel Extension}

The wheel extension is globally rigid.

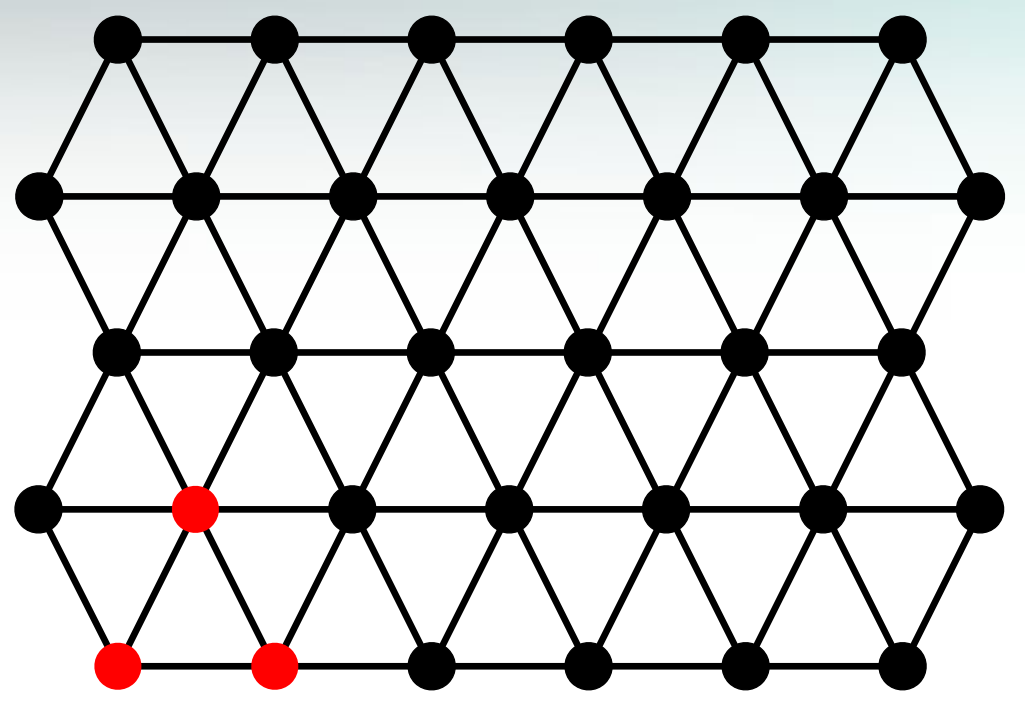

The above network is a wheel extension but NOT a trilateration extension. 


\section{WHEEL Protocol}

$\square$ Iterative localization

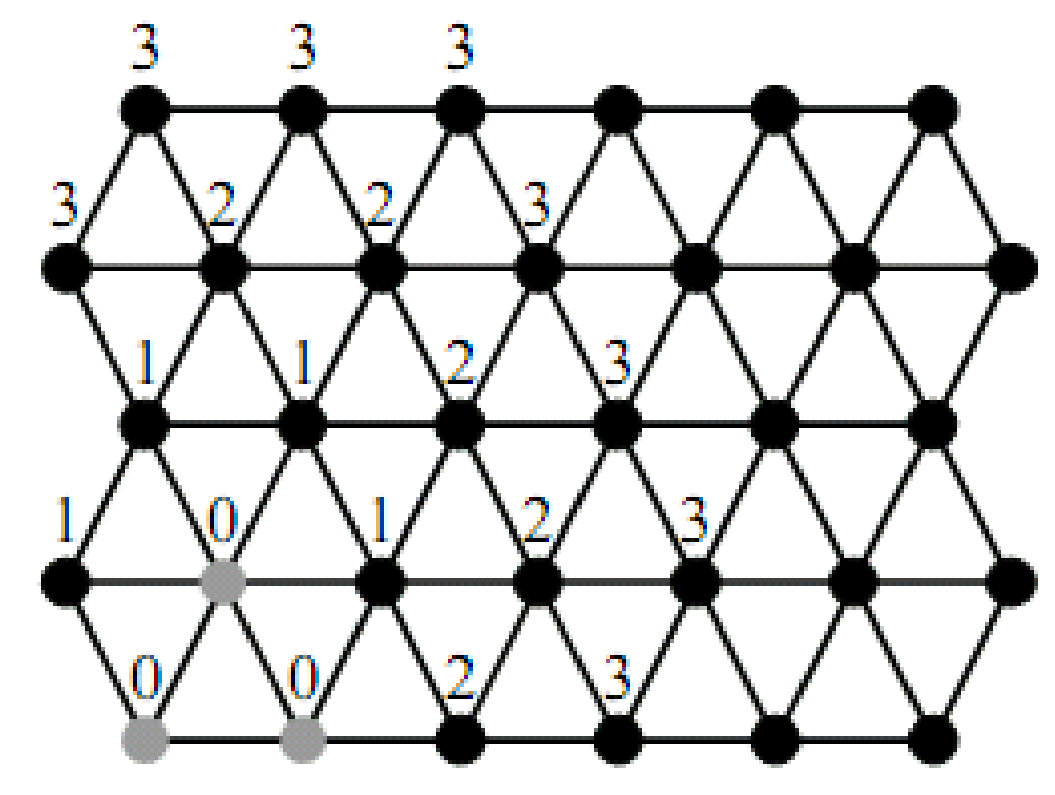




\section{Advantages of WHEEL}

$\square$ Efficiency

- $\mathrm{O}(\mathrm{n})$ for ad-hoc networks

- $\mathrm{n}$ : the network size

$\square$ Low cost

- no extra cost compared with TRI

\section{$\square$ Optimality
Optimal among ALL algorith
use only 1-hop information. \\ $\square$ Optimality
Optimal among ALL algorithms that
use only 1-hop information. \\ $\square$ Optimality
Optimal among ALL algorith
use only 1-hop information.}

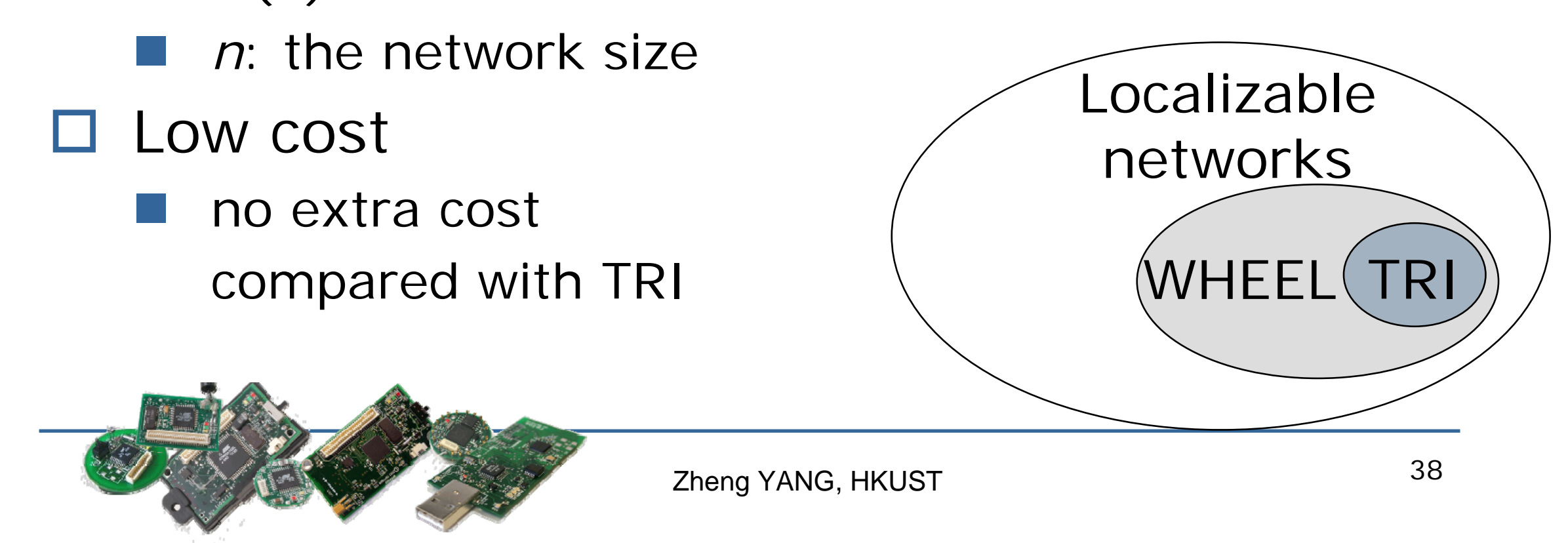




\section{Advantages of WHEEL}

$\square$ Using WHEEL, node 1 and 2 can be identified as localizable

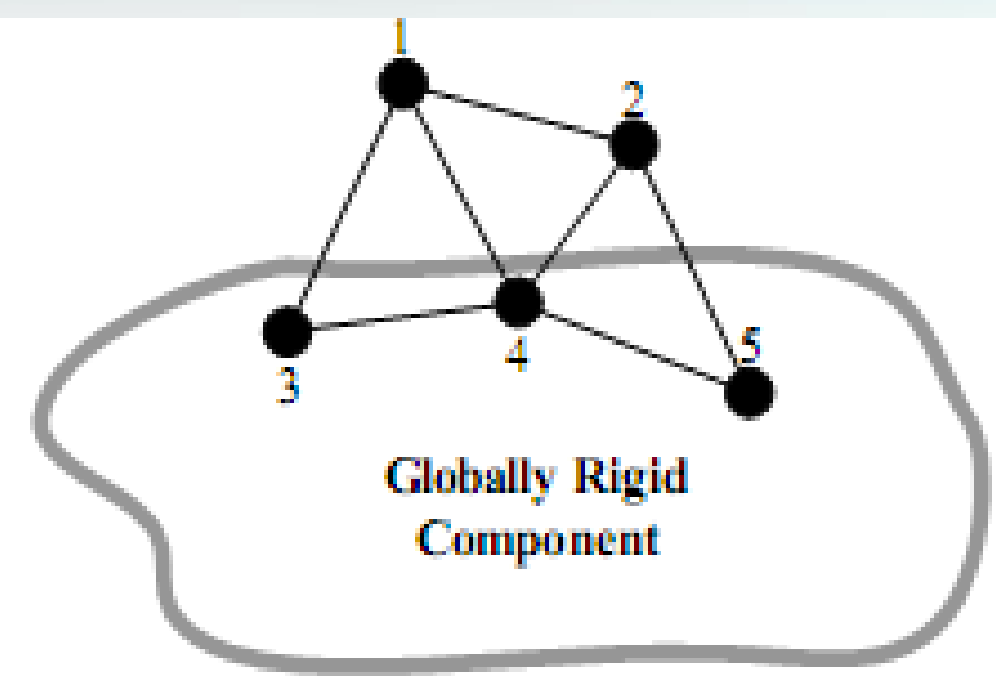




\section{Outline}

$\square$

$\square$ Localization

$\square$ Localizability

- Network Localizability

- Distributed Localizability Testing

- Node Localizability

$\square$ Conclusion and Future Study 


\section{Node Localizability}

\section{Observations}

from a working WSN: OceanSense

Almost all the time the network is NOT entirely localizable.

A large portion, on average nearly $\mathbf{8 0 \%}$, of nodes are actually localizable. 


\section{Node Localizability}

$\square$ Node localizability

- To answer the question that whether a particular node has a unique location.

- Node localizability focuses on single node;

- Network localizability considers entire network 


\section{Why Is \\ Node Localizability I mportant?

$\square$ Partially localizable networks

- They are not localizable.

- A portion of nodes have unique locations while others do not.

$\square$ Application

- Some nodes draw remarkable attentions

$\square$ Abnormal readings

$\square$ Border area
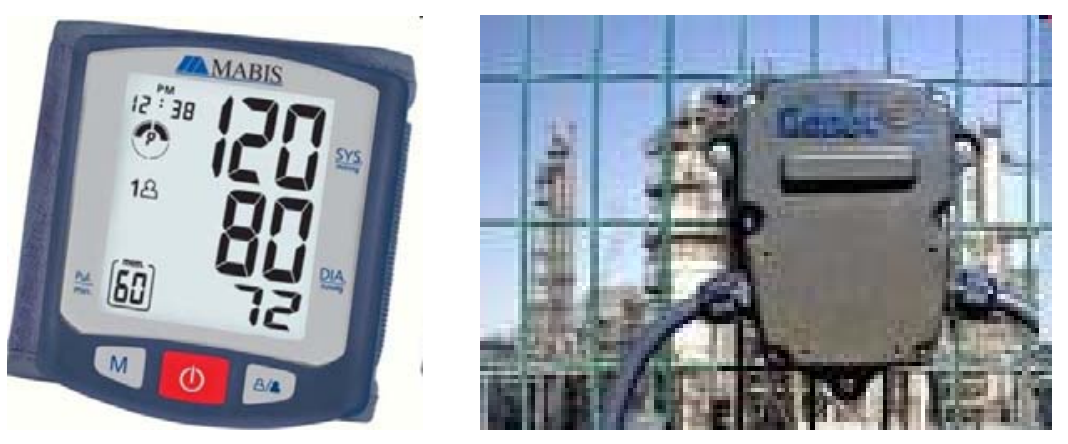


\section{Node Localizability}

$\square$ Which one is harder?
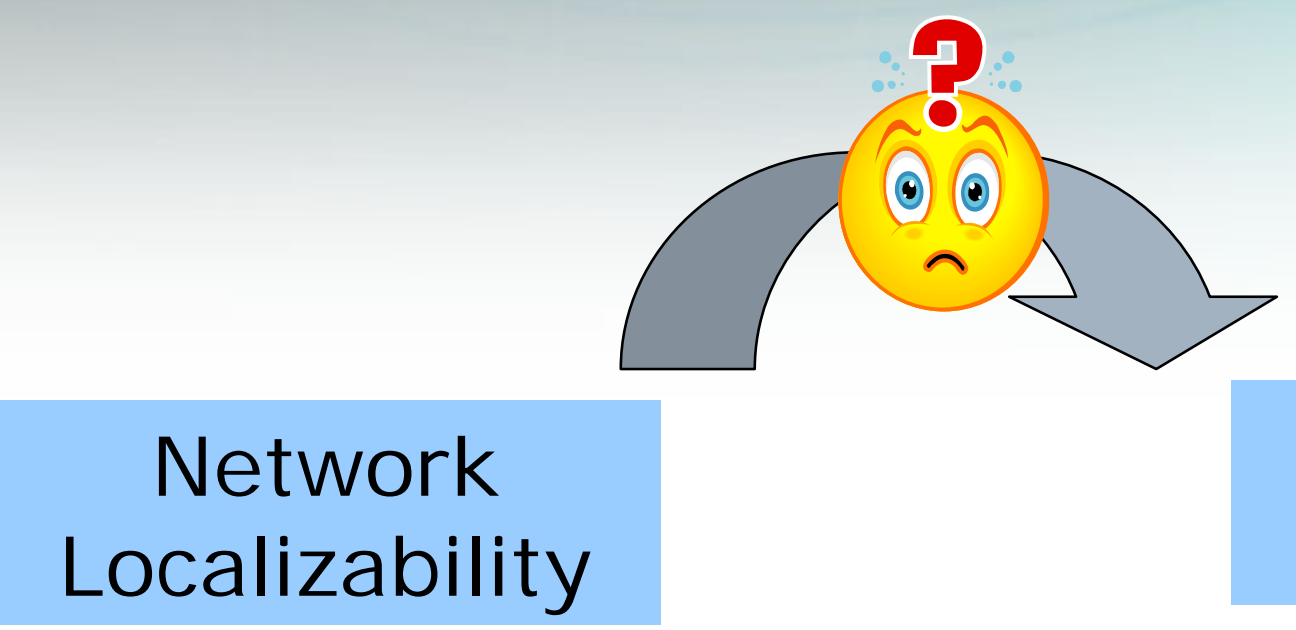

Node

Localizability

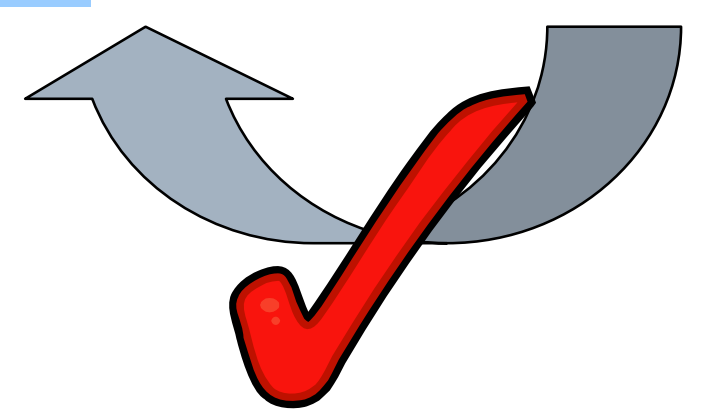




\section{Why Node Localizability Difficult?}

$\square$ A straight-forward solution (RR3B)

- Find a sub-network that is localizable

- Identify all nodes in the sub-network localizable

$\square$ Correct? YES, BUT ... 


\section{Why Node Localizability Difficult?}

$\square$ Missing localizable nodes

- $G$ is not 3-connected

- $u$ does not satisfy RR3B

$u$ is localizable

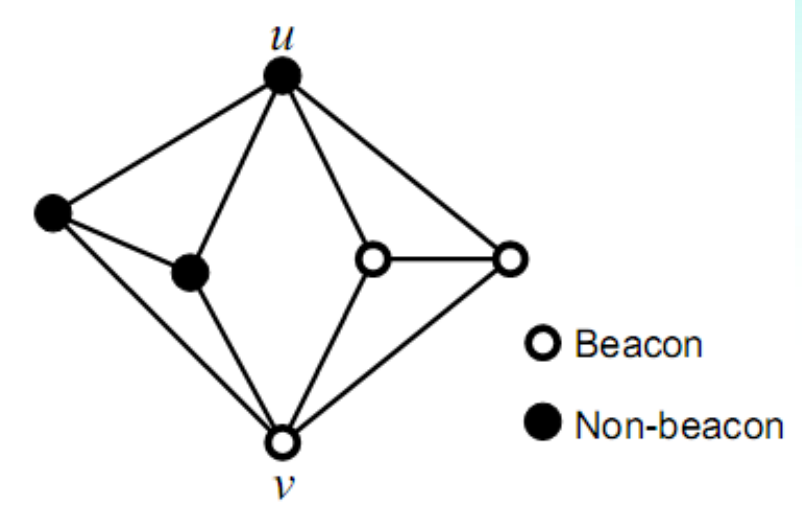

Some conditions essential to network localizability are no longer necessary for node localizability. 


\section{Conditions of Node Localizability}

$\square$ Necessity

Degree $=3$

- 3 vertex-disjoint paths to 3 distinct beacons [Goldenberg, 2005]

$\square$ Sufficiency

- Trilateration

- Localizable sub-network (RRT-3B) [Goldenberg, 2005] .

- Implicit edge [Eren, 2005] 


\section{Previous work}

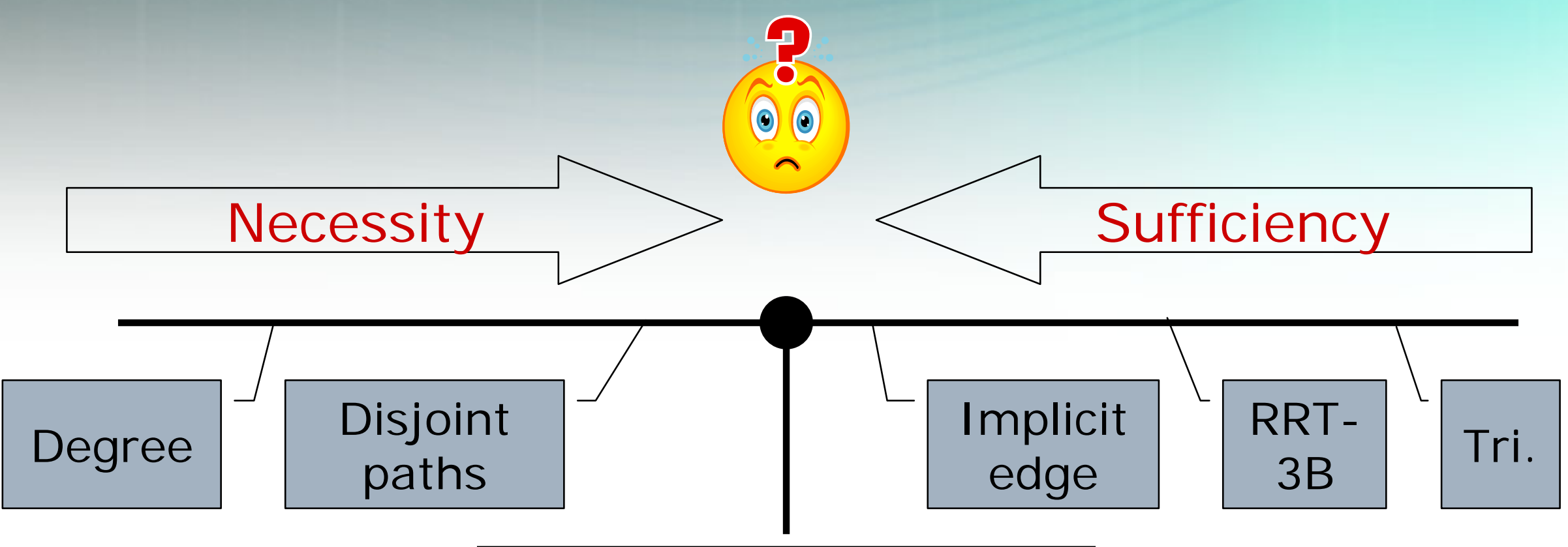

\section{Sufficient and Necessary condition}




\section{Necessary Conditions}

$\square 3$ vertex-disjoint paths (3P)

- Goldenberg et al., 2005

$\square$ Redundant Rigidity (RR)

- Yang et al., 2010

- If a vertex is localizable, it is included in the redundantly rigid component of beacon nodes. 


\section{Necessary Conditions}

\section{$\square$ Necessity}

- The combination of 2 necessary conditions is also a necessary condition

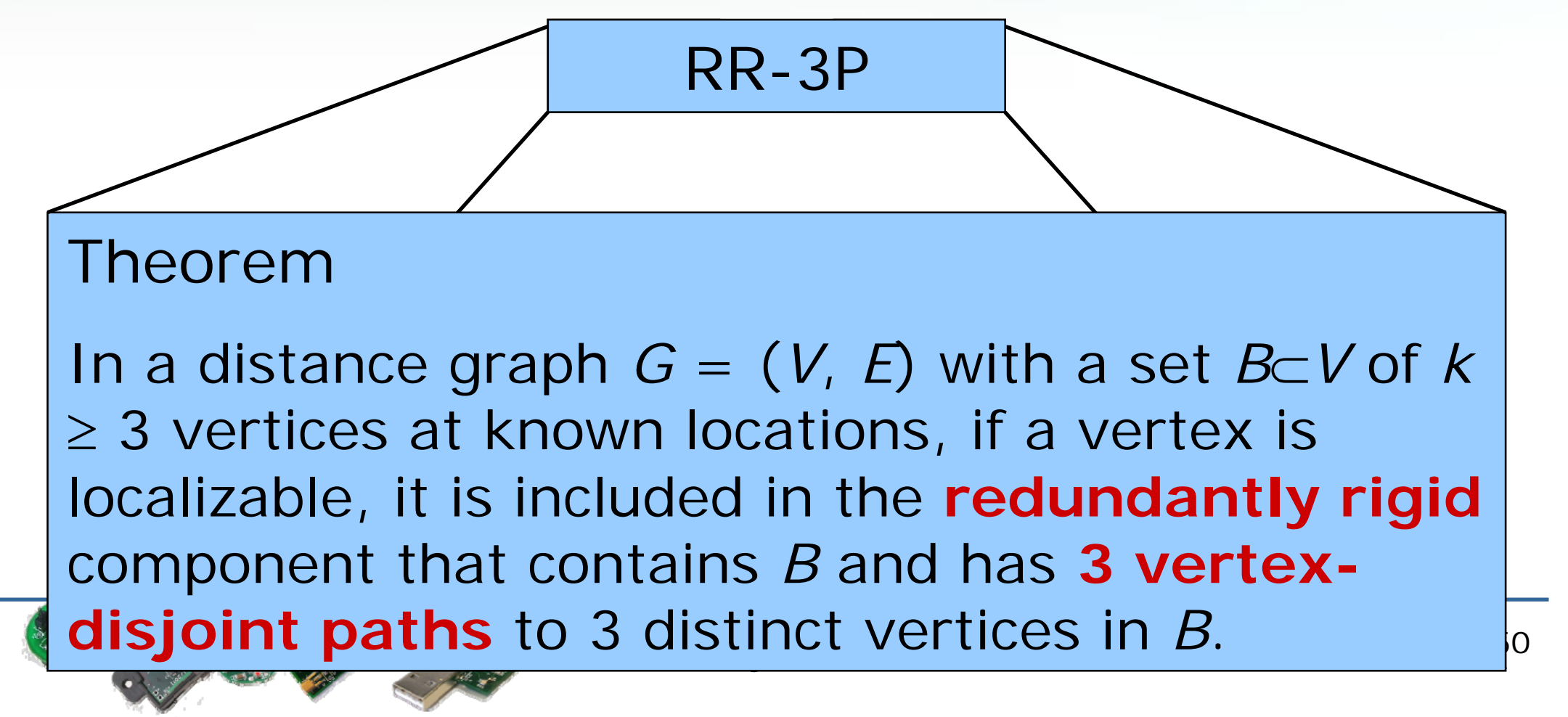




\section{Necessary Conditions}

\section{$\square$ RR-3P is NOT sufficient}

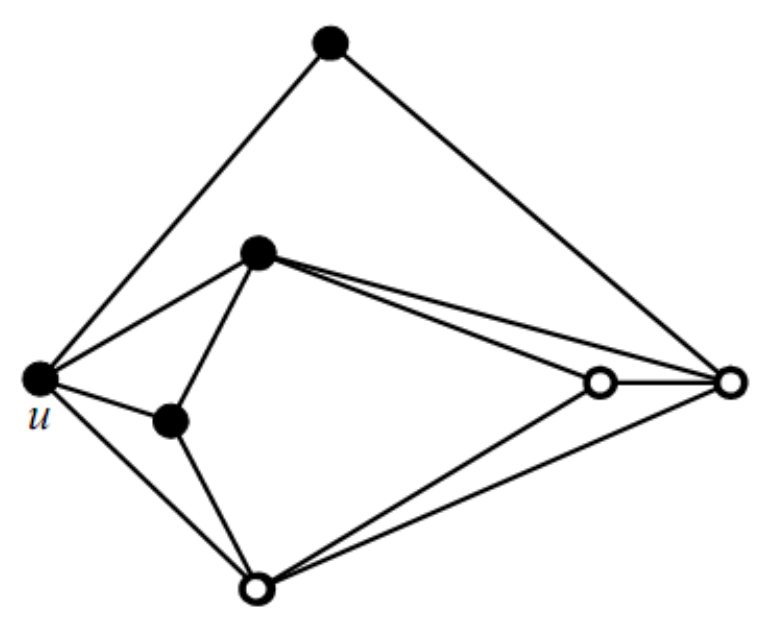

(a) The vertex $u$ satisfies RR-3P.

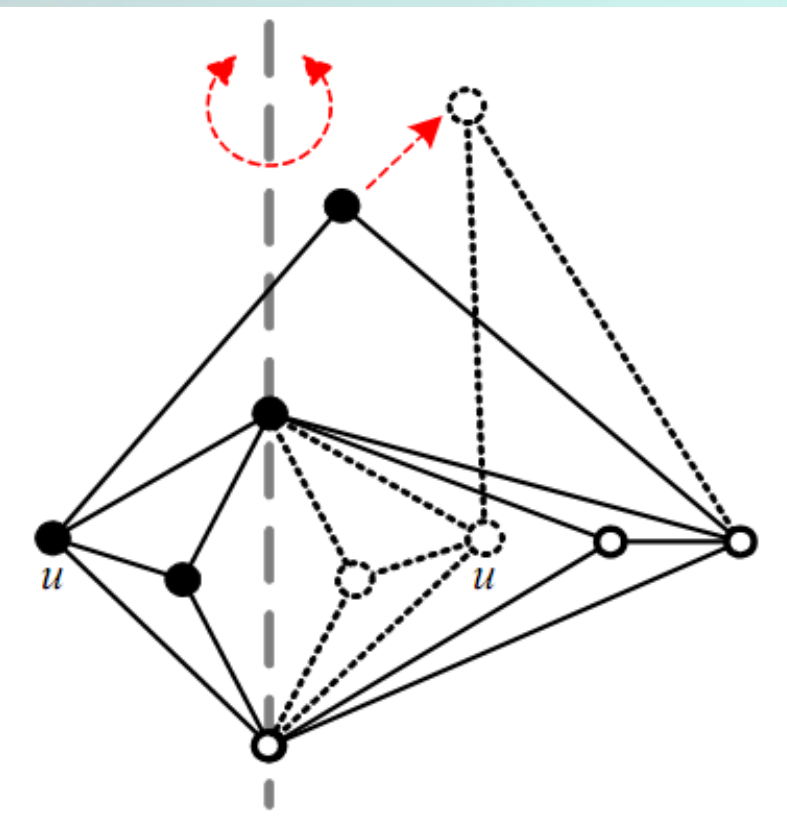

(b) $u$ suffers a discontinuous flexing. 


\section{Sufficient Conditions}

\section{$\square$ RR3P}

- All 3 paths are inside the RR component

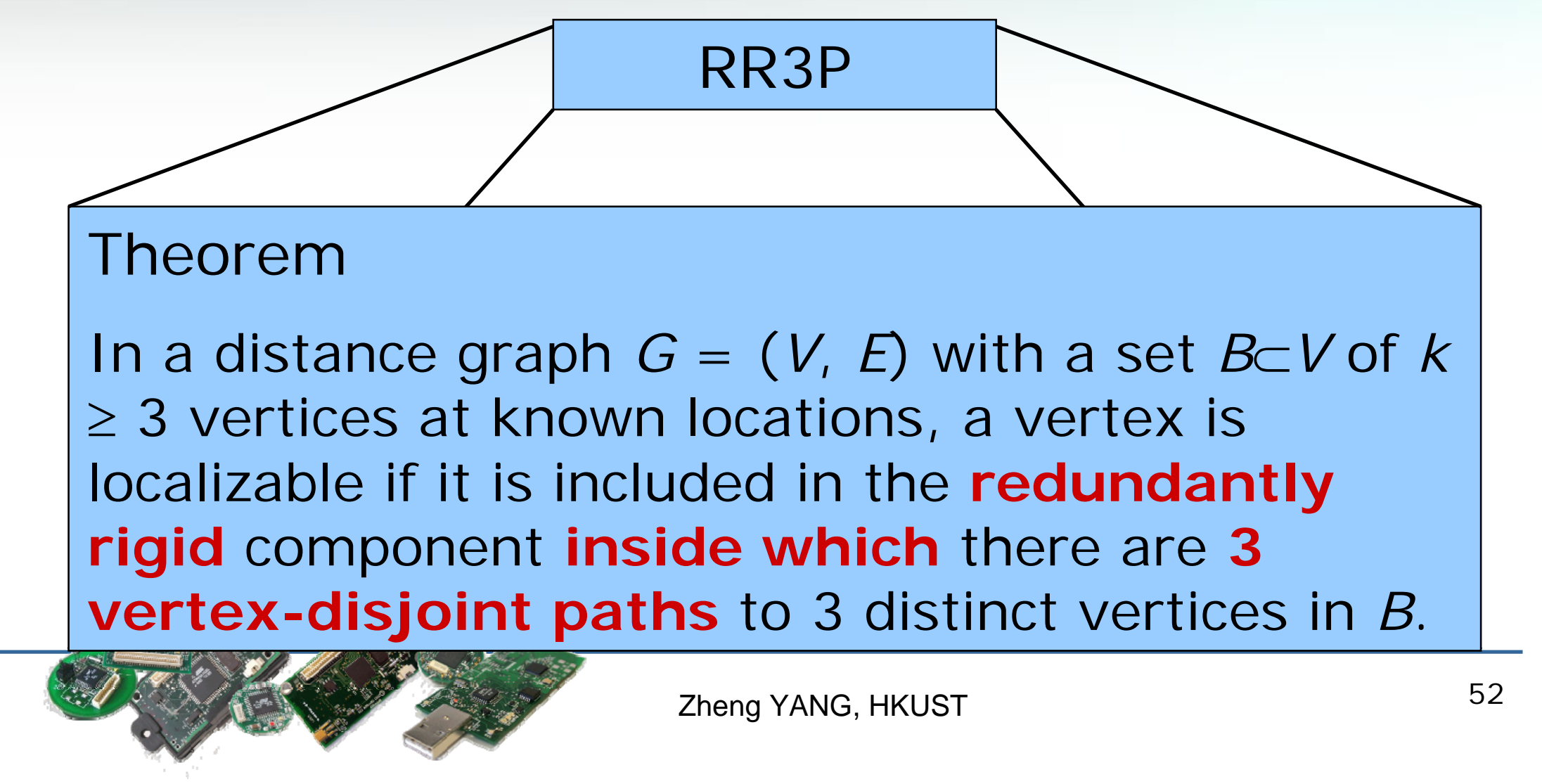




\section{Summary (1)}

\section{$\square$ Difference between RR-3P and RR3P}
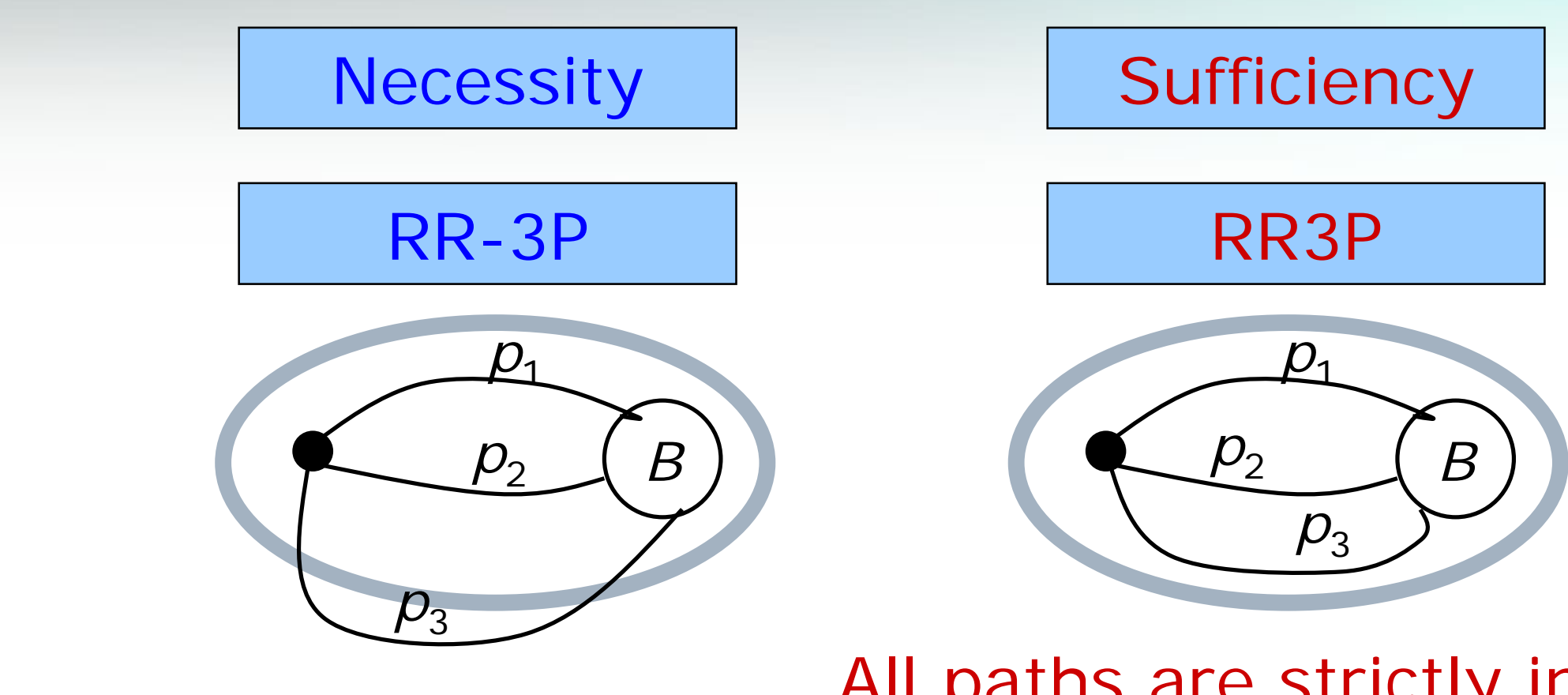

All paths are strictly included 


\section{Summary (2)}

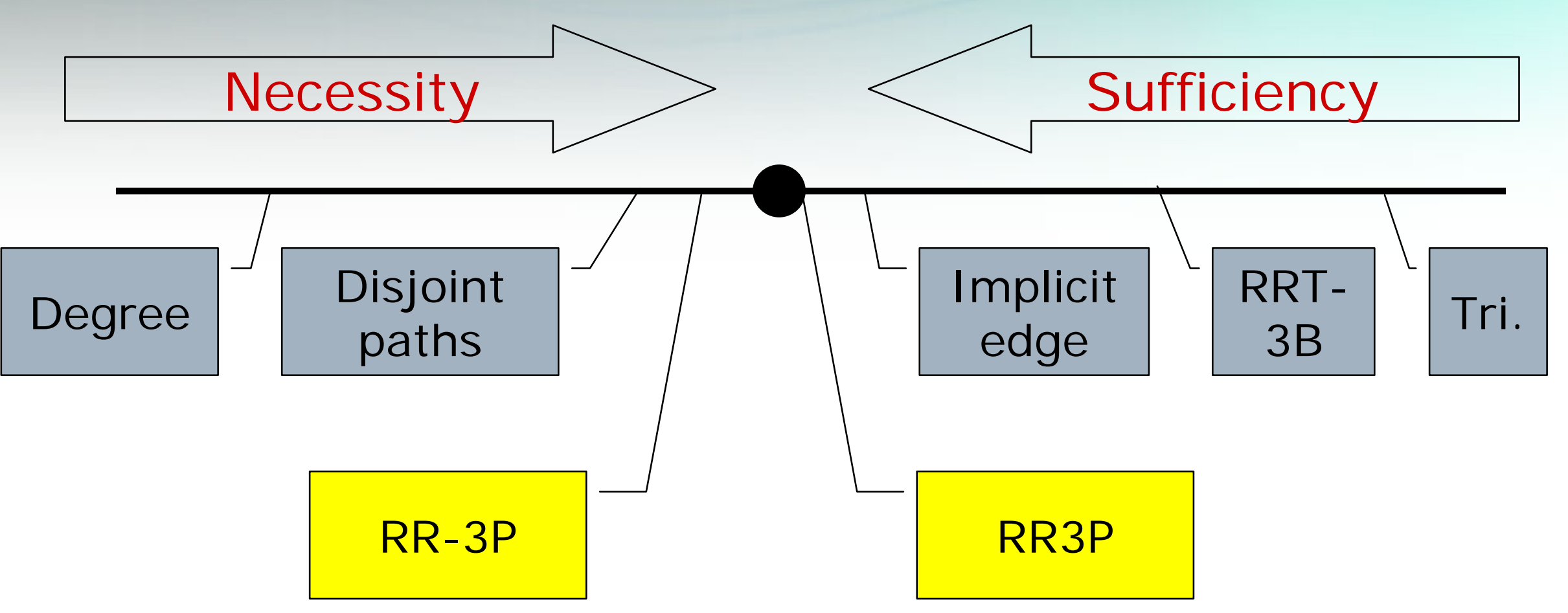




\section{Testbed experiment}

$\square$ OceanSense: A wireless sensor network for ocean monitoring

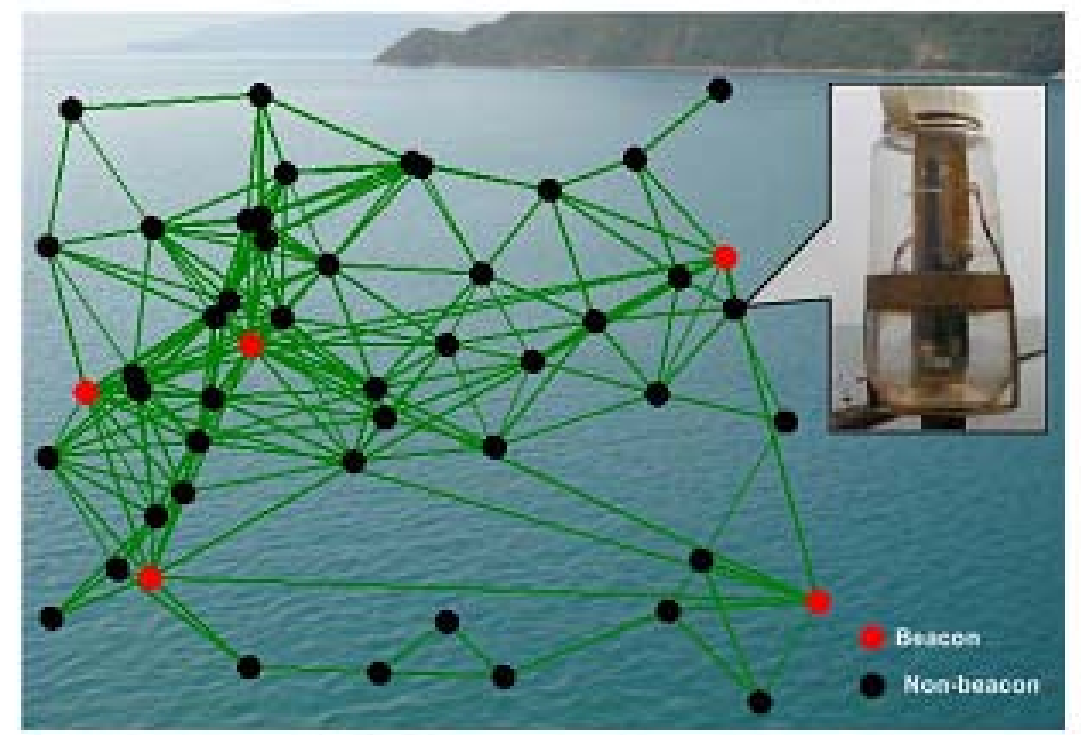

Figure 12: System Deployment. The upper right figure shows an encapsulated waterproof sensor mote. 
Testbed experiment

- Ocean monitoring

- 100+ sensors on sea surface

- Environmental data

$\square$ Temperature

$\square$ Humidity

$\square$ Ambient illumination

$\square$ Sea depth

- Restricted mobility

\section{$\square$ OceanSense}




\section{Testbed experiment}

$\square$ Sensor node platform: TelosB

- Long lifetime

- Low power

- Low cost

- Robust

- Standard compliant

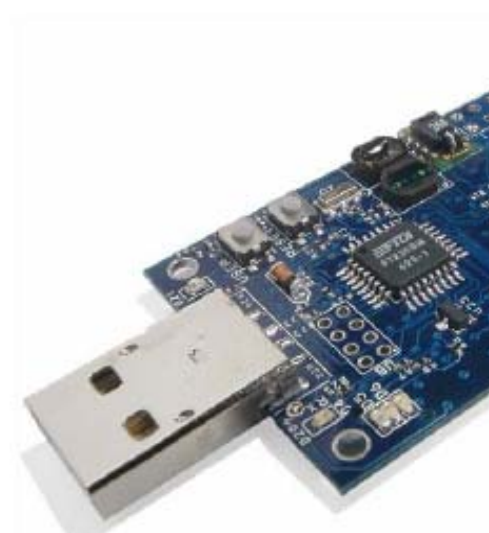




\section{TelosB Mote}

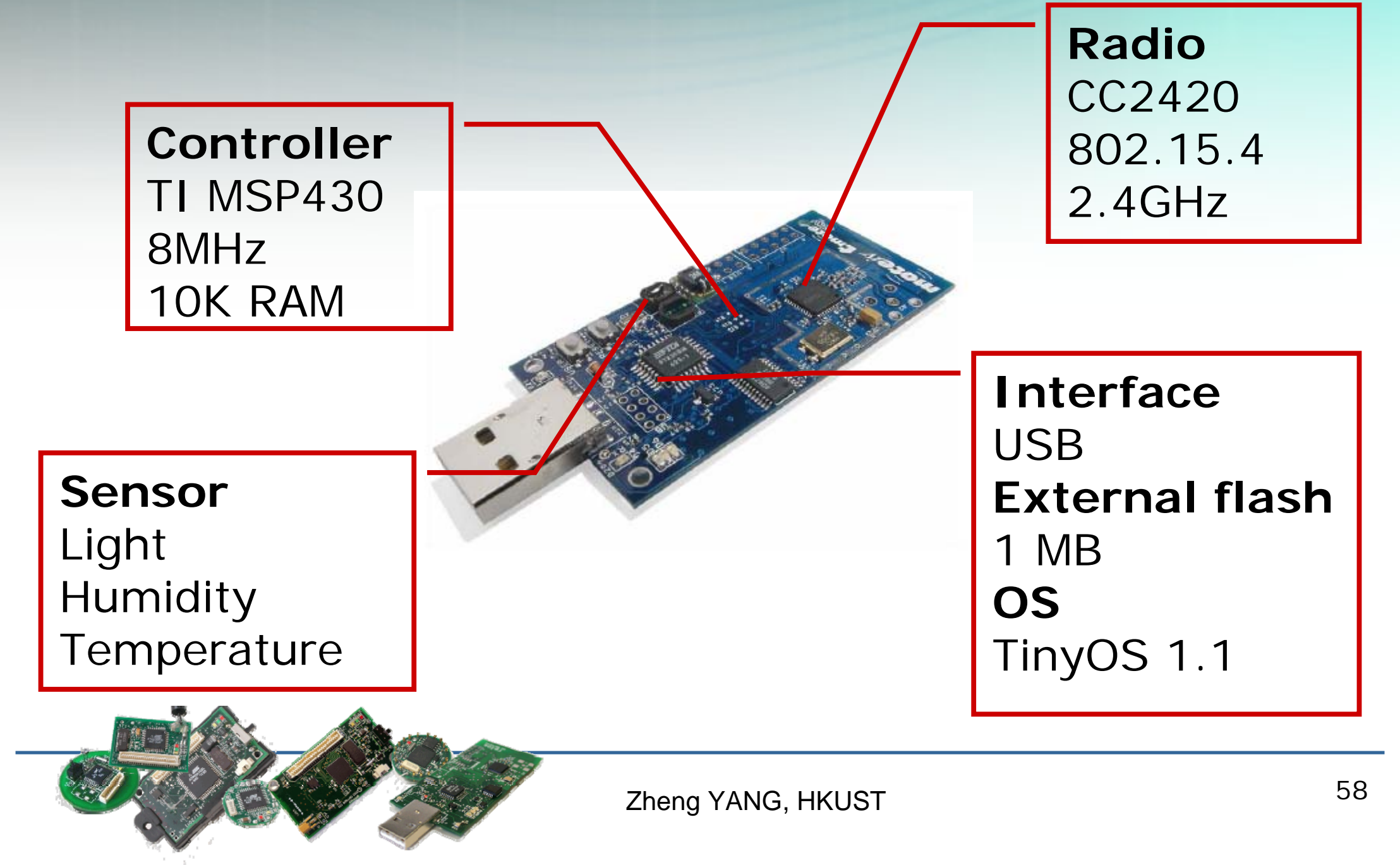




\section{Testbed experiment}

$\square$ Transceiver: Chipcon CC2420

- 802.15.4 compliant

- RF power: $-24 \mathrm{dBm}$ to $0 \mathrm{dBm}$

- Receiving sensitivity: $-94 \mathrm{dBm}$

- Outdoor range: $75 \mathrm{~m}$ to $100 \mathrm{~m}$

- Indoor range: $20 \mathrm{~m}$ to $30 \mathrm{~m}$

ZigBee $^{m}$

E Anipron

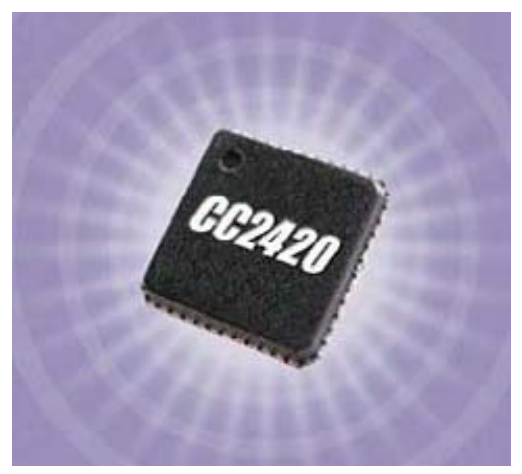




\section{ZigBee/802.15.4}

$\square$ ZigBee is suitable for wireless sensor networks

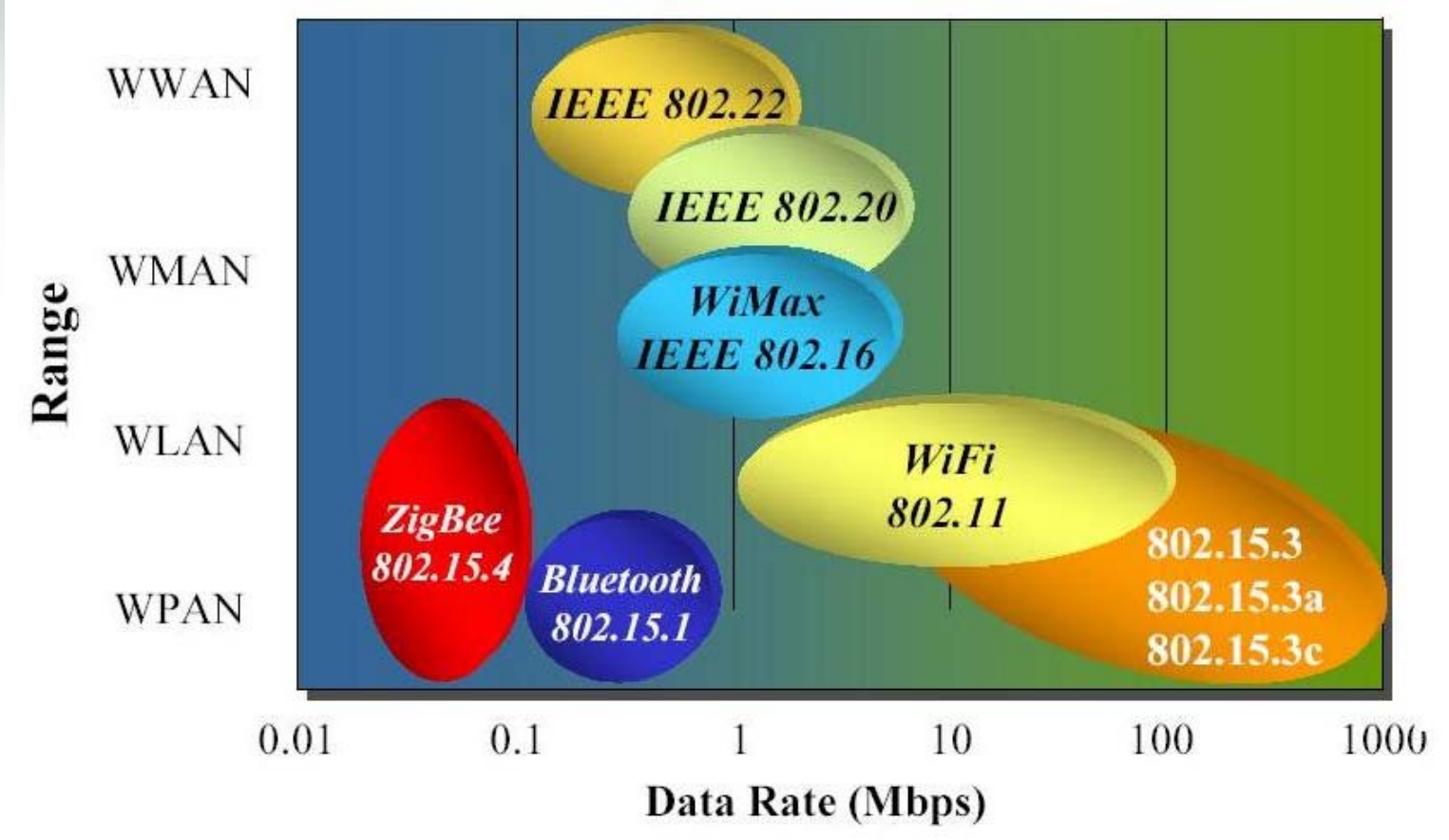




\section{Testbed experiment}

$\square$ All non-beacon nodes are identified as either localizable (black) or non-localizable (red).

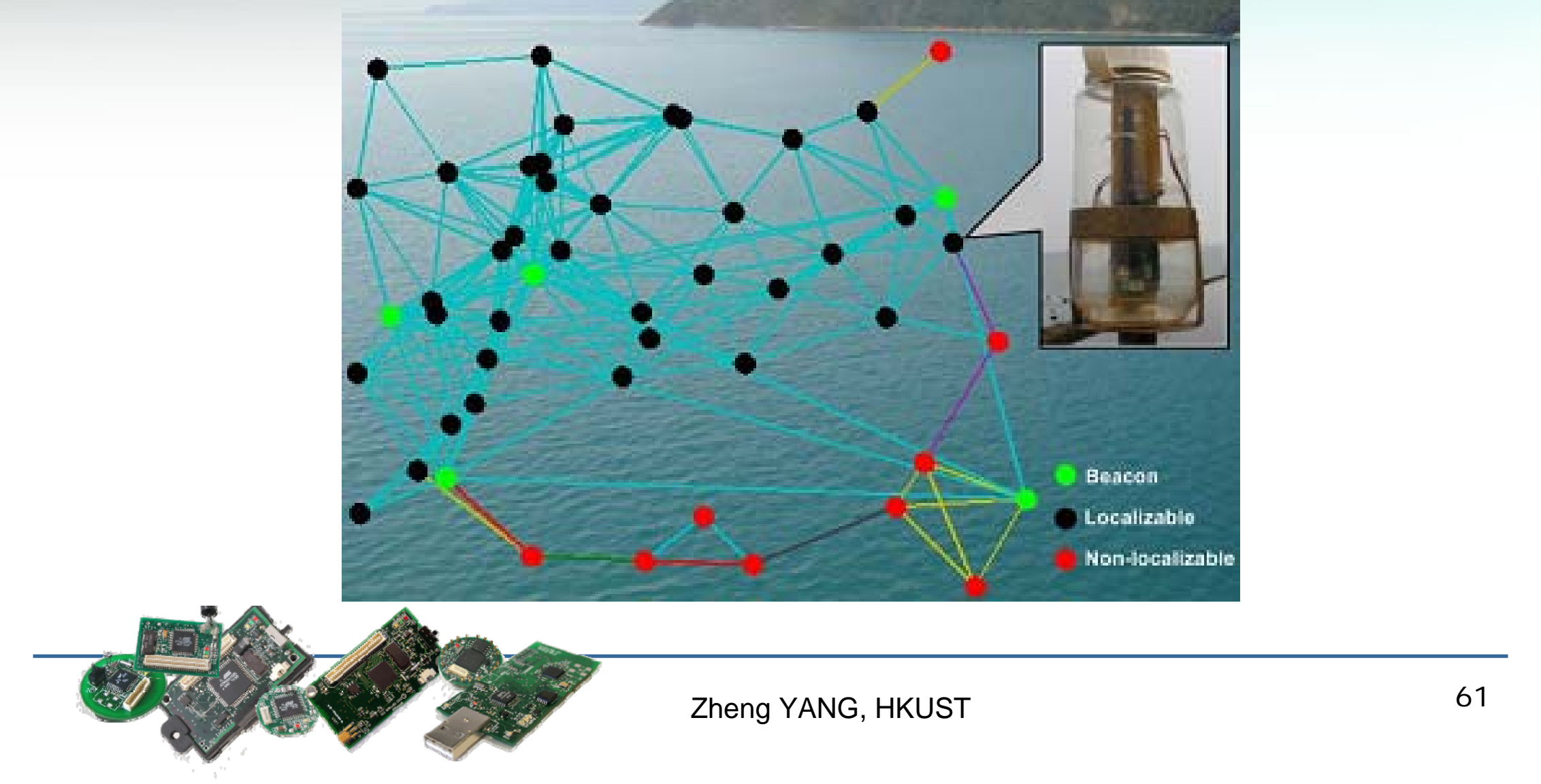




\section{Testbed experiment}

\section{$\square$ GreenOrbs}

- A WSN in a forest

- Long-term: over 1 year

- Large-scale: 1000 nodes
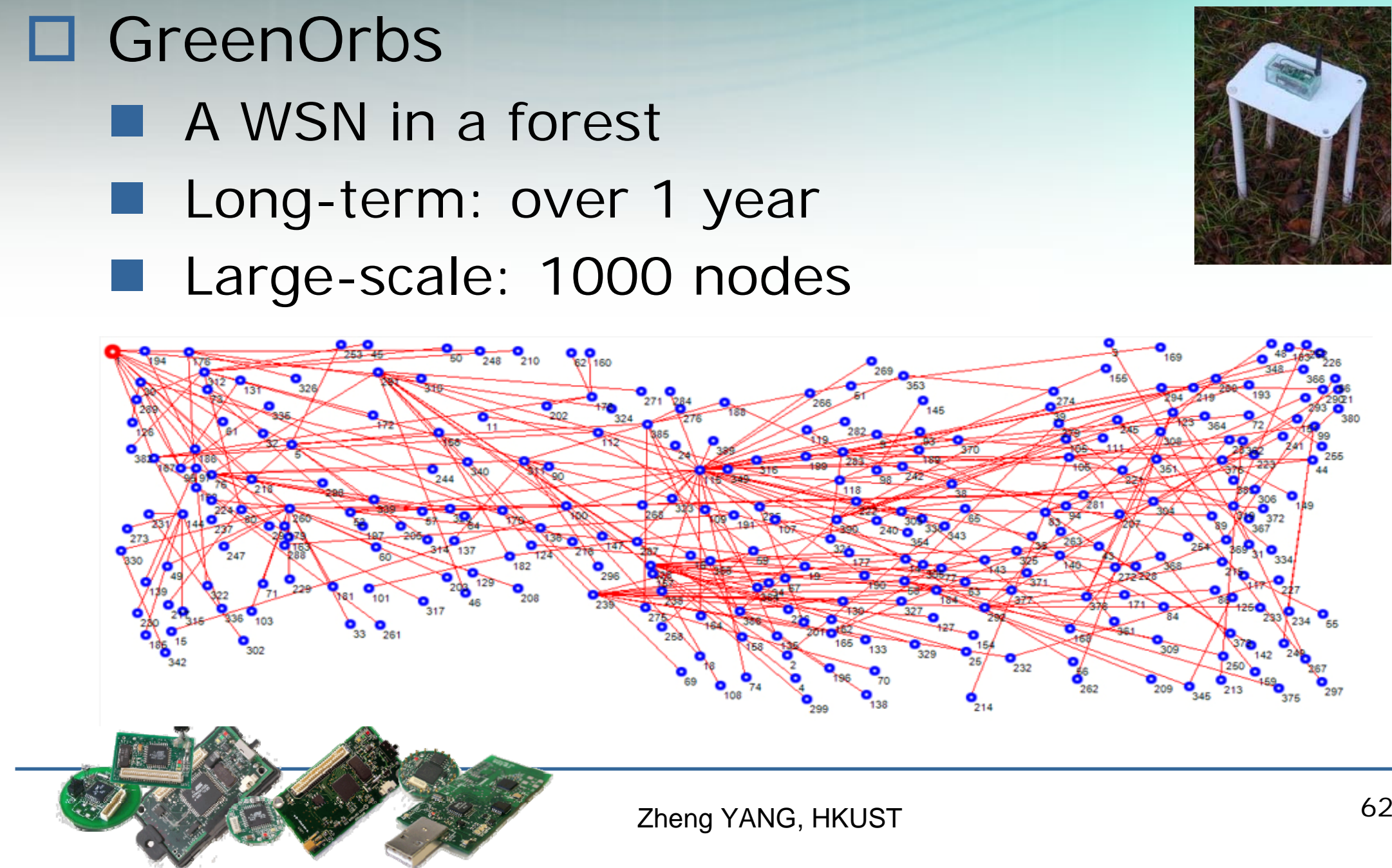


\section{Simulations}

$\square$ Metrics

- Number of nodes that can be identified (either localizable or non-localizable)

$\square$ Comparison

- Necessary conditions

$\square$ 3P V.S. RR-3P

- Sufficient conditions

$\square$ TRI V.S. RR3P 


\section{Simulation}

$\square$ RR-3P outperforms 3P; RR3P outperforms TRI

- Network with a "Z" hole

- Blue: non-localizable; Red: localizable; Grey:

unknown
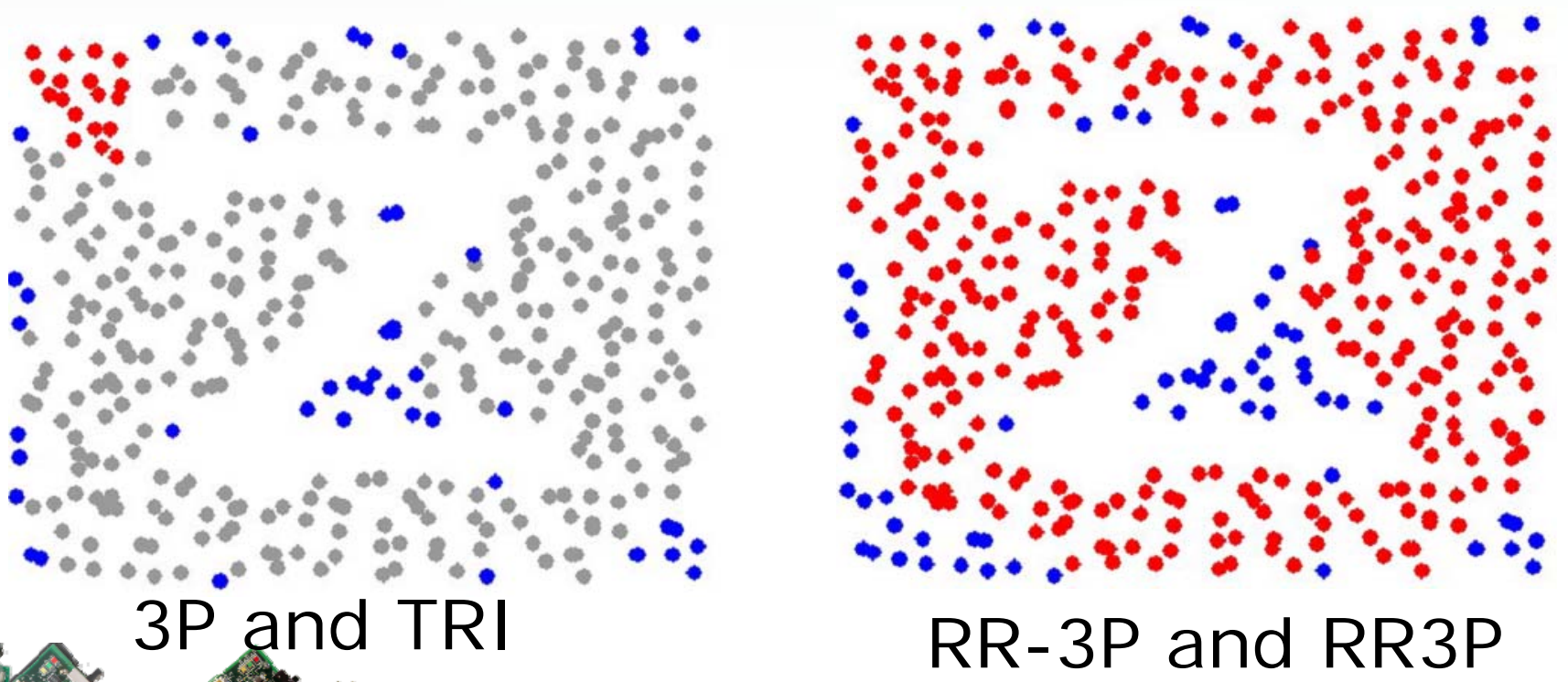


\section{Simulation}

$\square$ We conduct more simulations and the results are consistent.

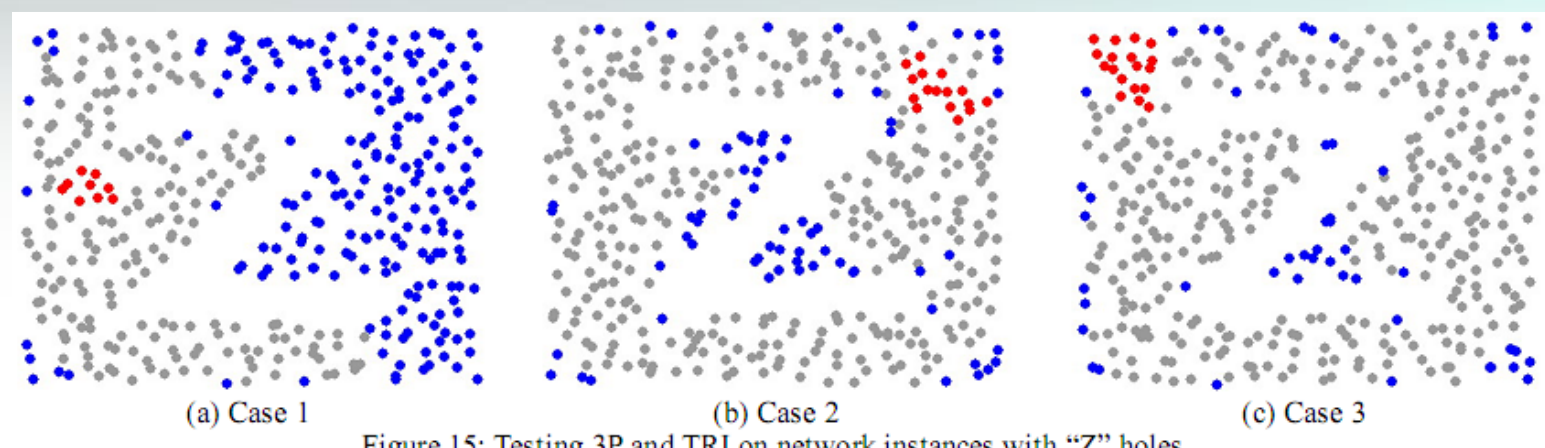

Figure 15: Testing 3P and TRI on network instances with " $\mathrm{Z}$ " holes.
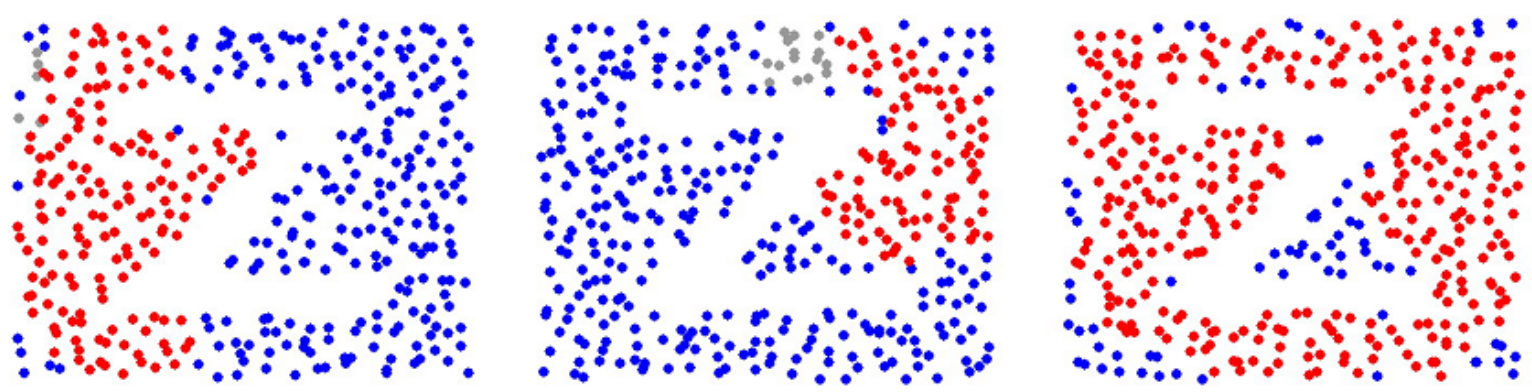

(a) Case 1

(b) Case 2

(c) Case 3

Figure 16: Testing RR-3P and RR3P on network instances with " $\mathrm{Z}$ " holes. 


\section{Outline}

$\square$

$\square$ Localization

$\square$ Localizability

- Network Localizability

- Distributed Localizability Testing

- Node Localizability

$\square$ Conclusion and Future Study 


\section{Conclusion}

$\square$ Distributed localizability testing

- We propose a new algorithm: WHEEL

- Efficiency: communication and computation

- Optimality: the best of all distributed approaches

- A nice substitute of TRI:

"All gains, no pains"

WHEEL

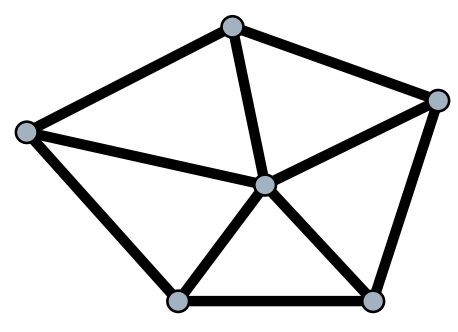




\section{Conclusion}

$\square$ Node localizability

- Analyze Limitations of network localizability

- Propose the concept of node localizability

- Derive necessary and sufficient conditions

Graph Rigidity

Theory

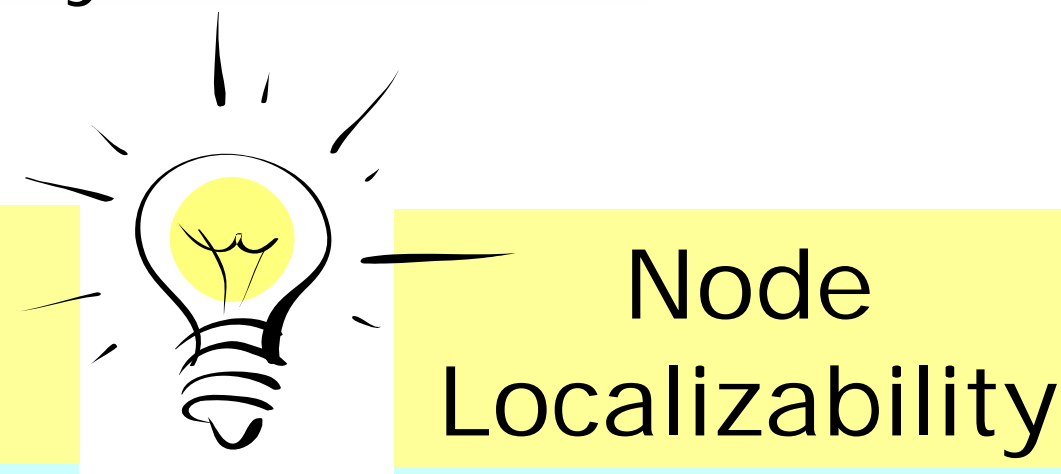

Application 


\section{Future Work}

$\square$ Research issues related to localizability

- Localizability under noisy ranging

- Localizability-aided localization

- Min cost localizable networks

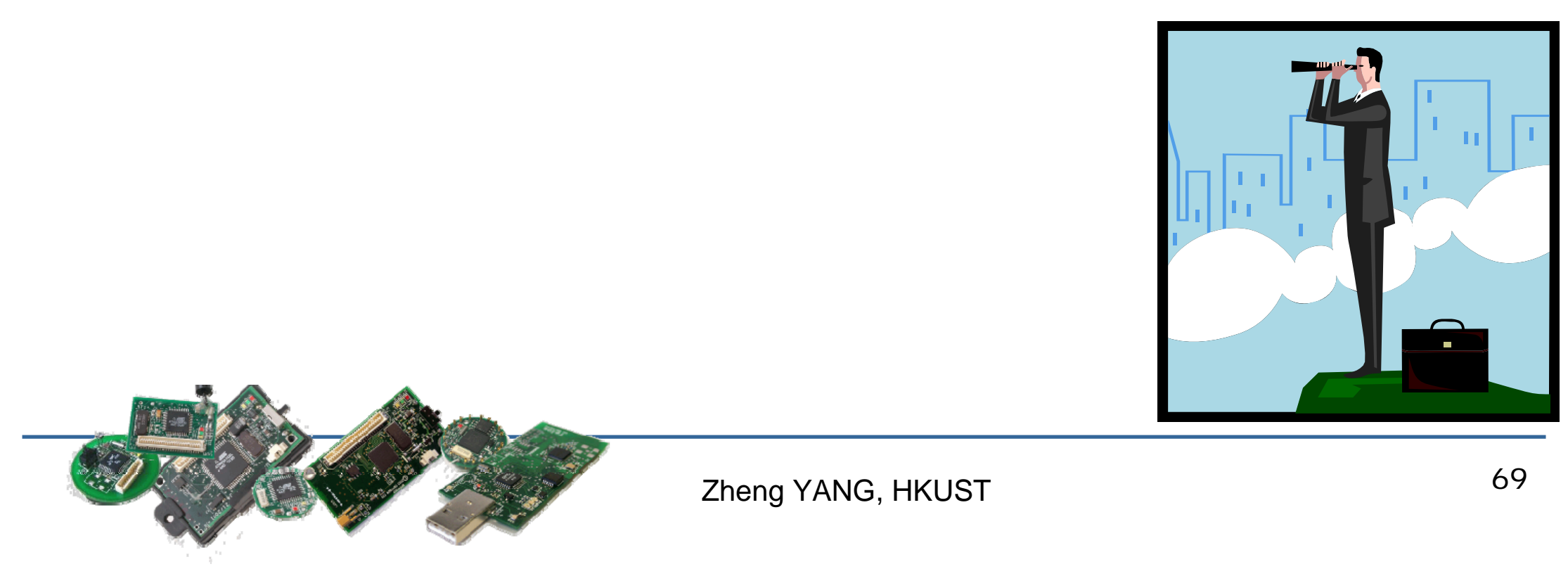




\section{Selected J ournal Publication}

Beyond Trilateration: On the Localizability of Wireless Ad-hoc Networks

Zheng Yang, Yunhao Liu, and Xiang-Yang Li

I EEE/ ACM Transactions on Networking (TON)

- Quality of Trilateration: Confidence based Iterative Localization Zheng Yang and Yunhao Liu

I EEE Trans. on Parallel and Distributed Systems (TPDS)

- Location, Localization, and Localizability Yunhao Liu, Zheng Yang, Xiaoping Wang, and Lirong Jian J ournal of Computer Science and Technology (J CST)

- Beyond Rigidity: Obtain Localizability with Noisy Ranging Measurement

Xiaoping Wang, Zheng Yang, Jun Luo, and Changxiang Shen International J ournal of Ad Hoc and Ubiquitous Computing (IJ AHUC) 


\section{Selected Conference Publication(}

Understanding Node Localizability of Wireless Ad-hoc Networks Zheng Yang and Yunhao Liu

\section{NFOCOM 2010}

- Beyond Trilateration: On the Localizability of Wireless Ad-hoc Networks Zheng Yang, Yunhao Liu, and Xiang-Yang Li

I NFOCOM 2009

- Quality of Trilateration: Confidence based Iterative Localization Zheng Yang and Yunhao Liu

\section{CDCS 2008}

- Sea Depth Measurement with Restricted Floating Sensors Zheng Yang, Mo Li, and Yunhao Liu,

\section{RTSS 2007}




\section{Acknowledgements}

$\square$ Thesis Defense Committee

- Advisor: Prof. Yunhao Liu

- Prof. Lionel Ni

- Prof. Qian Zhang

- Prof. Susheng Wang

- Prof. Xiaohua Jia (CityU of Hong Kong) 


\section{Acknowledgements}

\section{$\square$ Collaborators}

- I would like to thank all my collaborators, without whom this work would not have been possible

- Mo Li

- Jiliang Wang

- Lirong Jian

- Xiaoping Wang

- Junliang Liu

- Prof. Xiangyang Li 

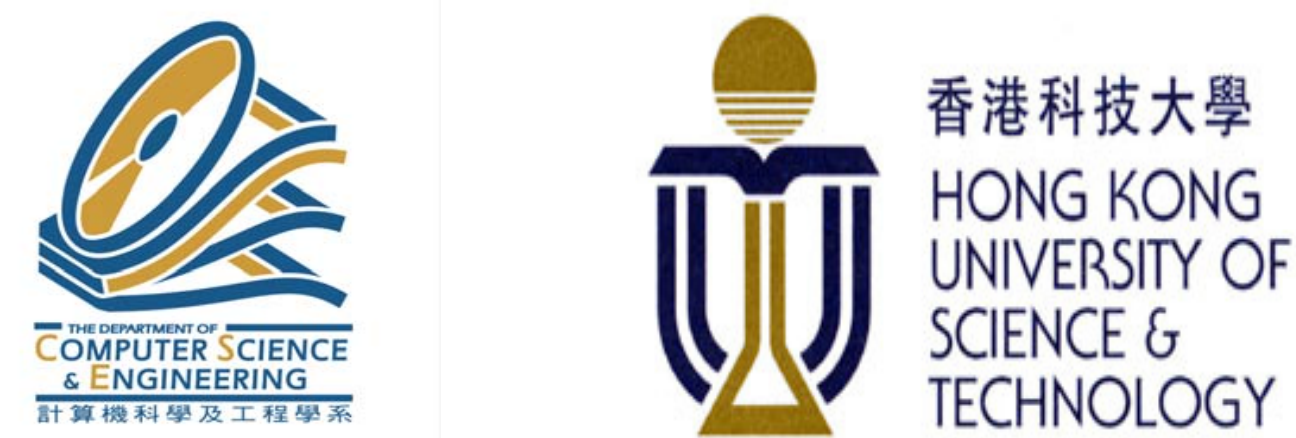

\section{Thank you.}

\section{Zheng Yang}

yangzh@cse.ust.hk

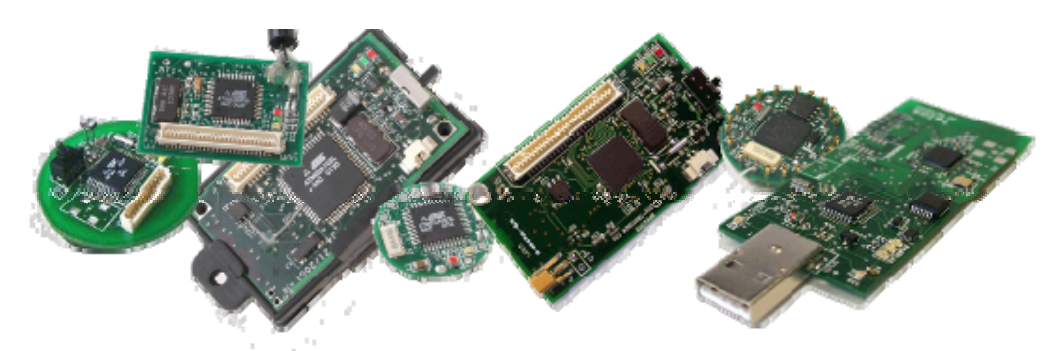

